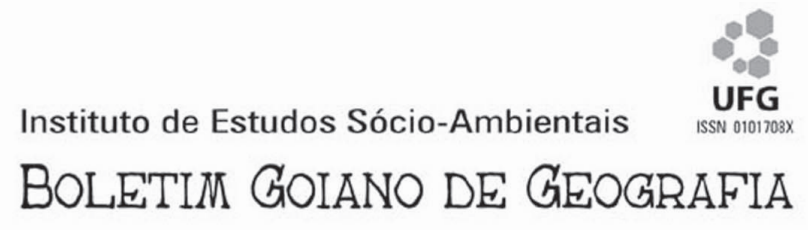

v. 26, n. 2, jul./dez. 2006

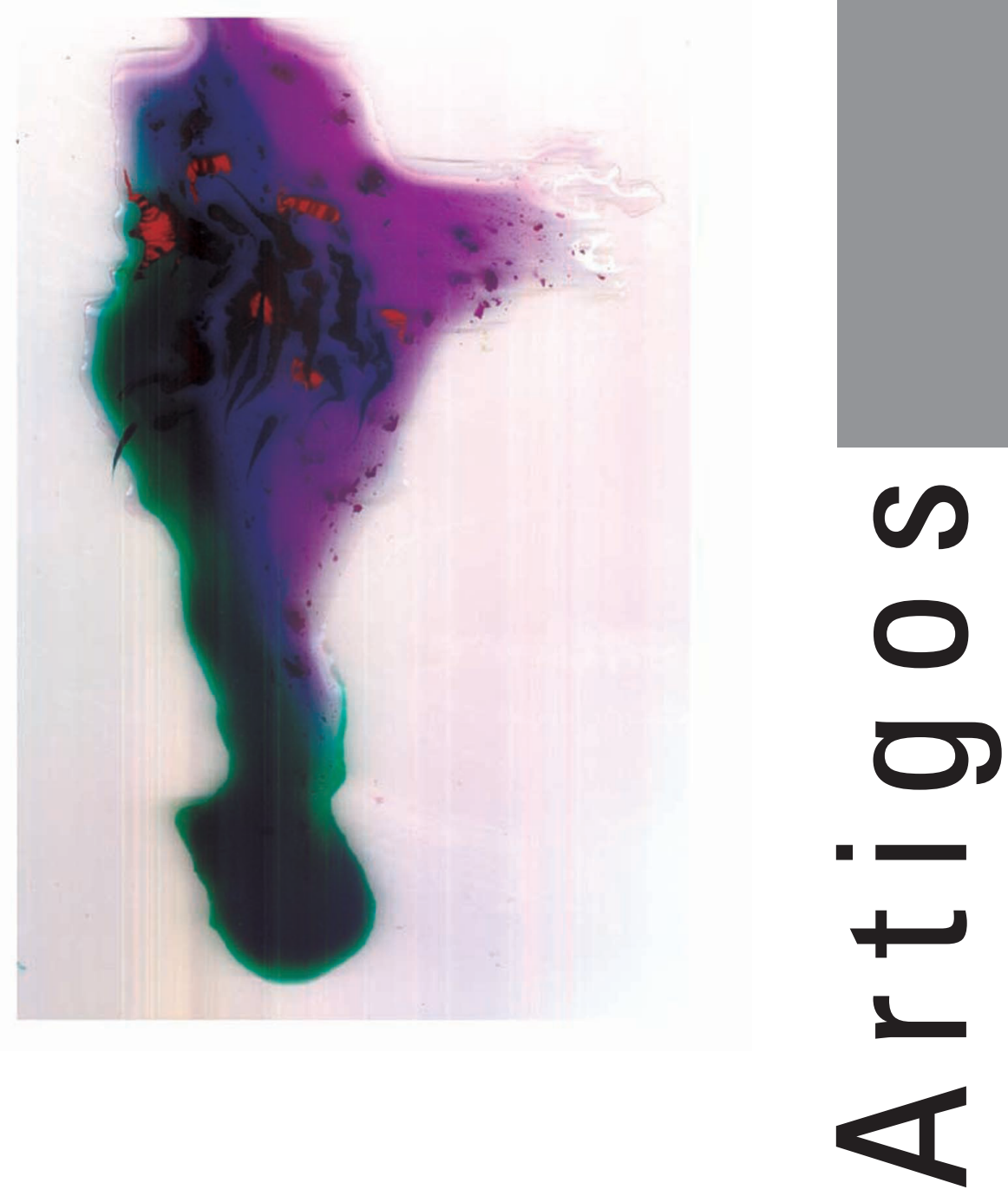




\title{
LES DIFFICULTES DE DEVELOPPEMENT DE L'ECOTOURISME DANS LES AIRES PROTEGEES DU CAMEROUN
}

\author{
LES DIFFICULTES DE DEVELOPPEMENT DE L'ECOTOURISME \\ DANS LES AIRES PROTEGEES DU CAMEROUN
}

\author{
Mesmin Tchindjang - Universite de Yaoundé I - Departament de geografie \\ mtchind@yahoo.fr \\ Samuel Aimé Abossolo - Universite de Yaoundé I - Departament de geografie \\ mtchind@yahoo.fr
}

Joseph Armathée - Ministère de l'enuirorment et de la protection de la nature joermathe@yahoo.fr

Vincent Francis Menga - Universite de Yaoundé I - Departament de geografie e_menga@yahoo.fr

\section{Résumé}

La création du premier parc national mondial le $1^{\text {er }}$ mars 1872 à Yellowstone aux Etats-Unis, suivie 26 ans plus tard par celle de la Sabie Game Reserve qui devint le Krüger National Park en Afrique du Sud, a ouvert la voie à la multiplication des aires protégées dans le monde entier. Le but de celles-ci est de préserver le potentiel naturel des différents pays et de promouvoir le développement durable par l'écotourisme. Cependant, le Cameroun a attendu 60 ans pour créer sa première réserve (1932) érigée 36 ans plus tard (1968) en parc national (le Parc de Waza). A ce jour, ce pays compte près de 77 zones protégées d'une superficie totale de 7,51 millions d'hectares, soit plus de $15 \%$ du territoire national. Dans l'ensemble, on dénombre 12 parcs et réserves de plus de 2,3 millions d'ha de superficie. Mais ceux-ci n'ont de parcs que de nom: en effet, ils bénéficient d'une attention faible de la part des services publics compétents. De plus, ceux qui sont en charge de la promotion de l'industrie touristique sont insuffisamment outillés ou qualifiés. Cet article vise à établir un diagnostic critique des principales difficultés du développement de l'écotourisme au Cameroun dans les aires protégées.

Mots-clés: aire protégée, biodiversité, Cameroun, écotourisme, parc national, réserve, tourisme.

\section{Abstract}

The objective of this paper would be to provide the historical circumstances of the creation of these reserves, their evolution and expectations, their ecological situation and the lessons to learn. The overall aim would be to examine the problems associated with their exploitation in order as to provide a panacea. Finally our approach also aims to show the conceptual framework difficulties in management of protected areas that justified decision makers' resistances to international laws and conventions. For, the increase of protected areas numbers is followed unfortunately by the decrease of biodiversity and other natural resources and also the decrease of tourist visits and incomes.

Key-words: biodiversity, Cameroon, ecotourism, national park, protected areas, reserve, tourism. 


\section{Introduction}

Le Cameroun dispose à ce jour de près de 20 aires protégées, dont des parcs nationaux, des réserves et des sanctuaires dont les atouts touristiques et écotouristiques sont indéniables. Cependant, 30ans après la création $\mathrm{du} 1^{\mathrm{er}}$ parc camerounais, le rôle des aires protégées sur le développement du tourisme et de l'écotourisme est allé décroissant dans tous les domaines alors que celui des parcs kenyans ne cesse de croître. Le Cameroun dispose pourtant de plus du double des parcs nationaux du Kenya et de ceux Français.

Pendant plus de 2 décennies, la valorisation touristique du patrimoine naturel n'était réalisée que par les 2 Provinces septentrionales (le Nord et l'Extrême Nord) ainsi que le Littoral à Douala. En Effet, 6 des 9 premiers parcs nationaux du Cameroun sont localisés dans le Grand Nord (Figure 1 et Tableau 1) du pays. Avec ce potentiel, le Cameroun apparaît comme le premier pays africain et devrait logiquement et normalement être ou devenir le premier pays en matière d'écotourisme en Afrique. Mais, il n'en est rien. Après 30 ans de tourisme timide et de tout tourisme avec sa kyrielle de blocages, de laxisme et d'antidotes inefficaces, une remise en question s'impose en même temps qu'une réorientation vers un tourisme efficace, écologiquement durable et rentable. Il s'agit de mettre en place un tourisme à composante vraiment environnementale qui intègre une concertation réelle avec les populations locales, leur formation, et la prise en compte de leur culture.

Le rôle des aires protégées et les difficultés de valorisation de ces sites écologiques 'biodiversifiés' assez bien répartis dans la quasi totalité des régions du Cameroun méritent d'être souligné. Après une présentation du contexte historique de la création de ces aires, de l'évolution et des attentes, nous aborderons la situation écologique; puis la fréquentation touristique. Nous terminerons par les difficultés de valorisation et les solutions

\section{Contexte Historique, évolution et attente}

\section{Le contexte historique}

L'histoire de la naissance des aires protégées dans le monde démarre dans l’Etat américain du Wyoming avec la création en 1872 du Parc National 
de Yellowstone. Sur le Continent Africain la toute première aire est la Sabie Game Reserve de l'Afrique du Sud qui devint en 1898 le Krüger National Parc. Depuis ces deux dates mémorables, les Puissances coloniales ont érigés dans leur territoire de dépendance respectif des parcs, réserves et jardins zoologique. Ainsi, en Afrique Noire francophone, les premiers sites protégés verront le jour en 1920. Mais le premier inventaire des aires protégées dans le monde ne paraîtra qu'en 1967, date à laquelle J.P. Harroy inventorie 1204 territoires protégés, Sournia (1998). Cet inventaire est suivi en 1974 par une nouvelle liste qui fait état de 1422 aires protégées. De nos jours, on en dénombre plus de 3000 sites protégés d'environ 600millions ha soit $3 \%$ des terres émergées de la Planète. L'Afrique garde la part belle, notamment l'Afrique Centrale avec 23 milions ha soit $6 \%$ de sa superficie, malheureusement avec seulement 32 millions de $\$$ de recettes.

Pour ce qui est du Cameroun, tout a démarré dans le Grand Nord du pays et les superficies des aires protégées n’ont cessé de s'accroître au point où les autorités veulent le porter de 15\% actuellement (4\% au début, 18\% d'ici 2010) à 30\% du territoire national. Tout a commencé bien timidement par le parc National de Waza qui est l'aire la plus fréquentée et la mieux connue. Le 12 juin et le 19 novembre1932 marquent la date de la création des premières réserves forestières camerounaises en l'occurrence celle de Mozogo Gokoro et celle de la Bénoué. Le 12 septembre 1933, le Dr Jeannin, Conservateur de la Nature et Chef des Services Vétérinaires de la Région du Nord présente au Haut Commissaire de la République Française au Cameroun un projet de création d'une $3^{\text {ème }}$ réserve en l'occurrence celle de Waza. L'acte de création est signé le 24 mars 1934 et la superficie initiale de 155000ha est portée 165000ha en 1935. Quatre objectifs guidaient l'Administration coloniale:

- Former un écran entre les troupeaux de bœufs de l'Extrême Nord non régulièrement vaccinés et ceux du Sud vaccinés contre la peste bovine qui pouvait infecter par contact les buffles et les bubales de la réserve.

- Après ce souci de protection, le second objectif était d'empêcher le trafic illégal des bœufs vers le Nigeria en détruisants les campements violants des trafiquants installés sur les bords des mares de la Réserve.

- Ensuite il s'agissait de favoriser le reboisement naturel et permettre l'exécution des travaux méthodiques de reboisement de la Réserve.

- Enfin, promouvoir le tourisme de vision en favorisant la multiplication et le rassemblement de grands mammifères de Waza. 
26, n. 2: 11-47, 2006

\section{Fig.1- LES AIRES PROTEGEES DU CAMEROUN}

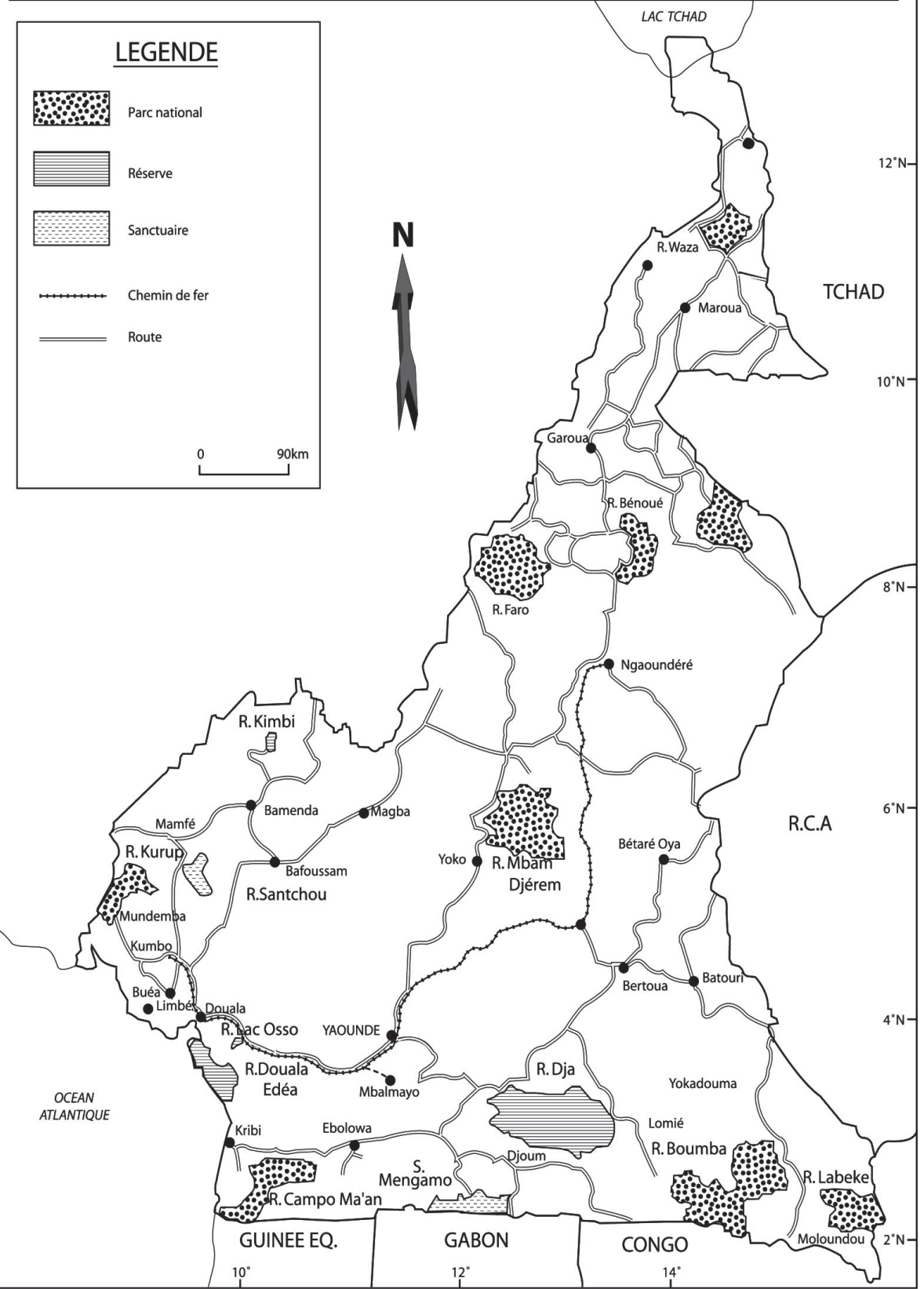


De ces quatre objectifs, les deux premiers ont été bel et bien satisfaits, mais les 2 derniers nous intéressent sur le plan écologique. Le second objectif est d'ailleurs l'objet d'une lutte sans merci de nos jours; le braconnage requérant l'implication des populations locales par une cogestion.

Tableau 1: Répartition régionale des aires protégées du Cameroun.

\begin{tabular}{|c|c|c|c|c|}
\hline Aires protégées & $\begin{array}{l}\text { Superficie } \\
\text { (ha) }\end{array}$ & Province & $\begin{array}{l}\text { Date création } \\
\text { Réserve/ZIC }\end{array}$ & $\begin{array}{c}\text { Date érection } \\
\text { en parc } \\
\text { national }\end{array}$ \\
\hline Parc National de Waza & 170.000 & Extrême Nord & 1932 & 1968 \\
\hline Parc National de Kalamaloué & 4500 & Extrême Nord & 1947 & 1972 \\
\hline Parc National de Mozogo-Gokoro & 1400 & Extrême Nord & 1932 & 1968 \\
\hline Parc National de Bénoué & 180.000 & Nord & 1932 & 1968 \\
\hline Parc National de Faro & 330.000 & Nord & 1932 & 1980 \\
\hline Parc National de Bouba - Ndjida & 220.000 & Nord & 1947 & 1968 \\
\hline Parc National de Korup & 126.000 & Sud Ouest & 1962 & 1982 \\
\hline Réserve du Dja & 526.000 & Est & 1950 & -- \\
\hline Réserve de Douala Edéa & 160.000 & Littoral & 1932 & -- \\
\hline Parc National de Campo Maan & 271.000 & Sud & 1932 & 2000 \\
\hline Réserve de Kalfou & 4.000 & Extrême Nord & 1933 & -- \\
\hline Réserve du Lake Ossa & 4.000 & Littoral & 1968 & -- \\
\hline Réserve de Kimbi & 5600 & Nord Ouest & 1964 & -- \\
\hline Réserve de Santchou & 7.000 & Ouest & 1968 & \\
\hline Réserve de Mbi Crater & 400 & Nord Ouest & 1964 & -- \\
\hline Sanctuaire de faune de Mengame & 17500 & Sud & -- & 2000 \\
\hline $\begin{array}{l}\text { Sanctuaire de faune de Mbayang } \\
\text { Mbo }\end{array}$ & 48500 & Sud Ouest & -- & 2000 \\
\hline $\begin{array}{l}\text { Parc National du Mbam et } \\
\text { Djérem }\end{array}$ & 416512 & $\begin{array}{l}\text { Centre et } \\
\text { Adamaoua }\end{array}$ & -- & 2000 \\
\hline Parc National de Boumba Bek & 210000 & Est & - & 2000 \\
\hline Parc National de Lobeke & 428000 & Est & -- & 2000 \\
\hline Zones d'intérêt cynégétique (28) & 2364396 & Nord et Adamaoua & 1968-1998 & -- \\
\hline Zones d’intérêt cynégétique (11) & 1213400 & Est (Moloundou) & $2000-2002$ & -- \\
\hline Zones d'intérêt cynégétique (01) & 75000 & Centre (Yoko) & 2002 & -- \\
\hline $\begin{array}{l}\text { Zones d'intérêt cynégétique à } \\
\text { gestion communautaire (14) }\end{array}$ & 993454 & Est (Moloundou) & $2000-2002$ & -- \\
\hline $\begin{array}{l}\text { Zones d'intérêt cynégétique à } \\
\text { gestion communautaire (02) }\end{array}$ & 89000 & Nord (Poli) & 2003 & -- \\
\hline
\end{tabular}

Source: MINFOF, 2005. 


\section{Evolution et attente sur les Aires Protégées}

Les enjeux écologiques du $21^{\text {ème }}$ siècle obligent la Communauté Internationale à serrer de près la notion de protection de l'environnement amorcée à Stockholm en 1972, poursuivie à Rio de Janeiro en 1992 et évaluée en Afrique du Sud en 2002. Dans le nouvel Ordre Ecologique qui émane $\mathrm{du}$ Sommet de Rio, un accent est mis sur la protection des pays abritant les espèces protégées et menacées de disparition par le biais des crédits et transferts de technologie. En vue du développement durable, les réserves de la Biosphère crées par L'UNESCO depuis 1976 ne cessent de se multiplier avec 4 visées fondamentales:

- Renforcer la conservation des ressources génétiques et des écosystèmes ainsi que la surveillance des espaces à haute valeur écologique.

- Entretenir la biodiversité animale et végétale.

- Associer concrètement la protection de l'environnement et la mise en valeur des ressources du territoire: tourisme, écotourisme pour un développement durable.

- Promouvoir les activités de recherche et d'éducation afin d'assurer le suivi scientifique.

Toutes ces actions sont étroitement liées. Cependant l'évolution des aires protégées au Cameroun ne semble pas tenir compte de ce plan à 4 volets: surveillance, entretien et développement, suivi scientifique et recherche. Il a fallu que les crédits de l'Agenda 21 soient déployés pour voir se mettre en place à Waza un produit écotouristique réussi associant:

- La gestion participative avec la réouverture des campements fermés

- La création des villages de culture à l'image de ceux existant en Afrique Australe

- Le début de zonage du parc avec une association des populations riveraines comme guide. Même ski des efforts restent à fournir, ces trois éléments concourent et font partie du développement durable. 


\section{Situation écologique du Cameroun et de ses aires protégées}

Le tableau nous présente la situation écologique des aires protégées camerounaises. Le pays étant marqué par une diversité impressionnante de relief et de climat (basses terres aux hautes terres), les sites protégées s'échelonnent du niveau de la mer jusqu'à $2000 \mathrm{~m}$ d'altitude sous des climats variés allant du climat équatorial au climat tropical. Il est vrai qu'au départ, les aires protégées étaient créées exclusivement dans les régions de savane; mais de nos jours au Cameroun, il en existe dans toutes les 10 provinces du pays avec leurs variantes topographiques, climatiques, hydrologiques et biologiques. Nous les avons classées dans le Tableau 2 suivant:

Tableau 2: Situation écologique des aires protégées au Cameroun.

\begin{tabular}{|c|c|c|c|c|c|}
\hline & $\begin{array}{l}\text { Sites à } \\
\text { l'interface } \\
\text { terre/mer: } \\
\text { 0-300m }\end{array}$ & $\begin{array}{c}\text { sites de basses } \\
\text { terres: } \\
50-300 \mathrm{~m}\end{array}$ & $\begin{array}{c}\text { Aires du } \\
\text { plateau Sud } \\
\text { Camerounais: } \\
\text { 700m }\end{array}$ & $\begin{array}{c}\text { Aires de } \\
\text { montagne et } \\
\text { plateaux: } \\
>1000 \mathrm{~m}\end{array}$ & $\begin{array}{l}\text { Aires } \\
\text { transfron- } \\
\text { talières }\end{array}$ \\
\hline Nombre & 03 & 10 & 08 & 08 & 08 \\
\hline Dénomination & $\begin{array}{l}\text { Campo-Ma'an, } \\
\text { Douala-Edéa, } \\
\text { Korup }\end{array}$ & $\begin{array}{l}\text { Waza, } \\
\text { Kalamaloué, } \\
\text { Kalfou, Campo- } \\
\text { Ma’an, Korup, } \\
\text { Lac Ossa, } \\
\text { Douala-Edéa, } \\
\text { Bénoué, Faro, } \\
\text { Bouba Ndjida. }\end{array}$ & $\begin{array}{l}\text { Dja, Lobéké, } \\
\text { Boumba Bek, } \\
\text { Mengame, Nki } \\
\text { Mbam Djerem, } \\
\text { Mpem Djim, } \\
\text { Sanaga Yong, } \\
\text { Lom Pangar }\end{array}$ & $\begin{array}{l}\text { Kimbi, Mozogo } \\
\text { Gokoro, } \\
\text { Bayang Mbo, } \\
\text { Mbi Crater, } \\
\text { Santchou, } \\
\text { Mbere valley, } \\
\text { Bakossi } \\
\text { Mountains, } \\
\text { Rumpi Hills }\end{array}$ & $\begin{array}{l}\text { Kalamaloué, } \\
\text { Bouba Ndjida, } \\
\text { Lobéké, Korup, } \\
\text { Boumba Bek, } \\
\text { Mengame, } \\
\text { Campo Ma’an, } \\
\text { Faro. }\end{array}$ \\
\hline
\end{tabular}

Ces sites classés sont conçus pour durer et laisser à des générations futures des traces d'un patrimoine écologique d'équilibre précaire. Ces héritages méritent à juste titre une utilisation rationnelle pour prétendre à un quelconque maintien. En effet, ce sont des souvenirs et des symboles irremplaçables et ceux qui les observent aujourd'hui doivent "bénir le souvenir de ceux qui ont créé ces patrimoines irremplaçable parce que l'on ne pourra plus jamais les constituer ». On peut seulement le conserver en l'aménageant. Cette préoccupation suppose et impose la création d'une banque de données pour stocker, classer, repérer, traiter et conserver minutieusement les caractérisations dynamiques ainsi que 
l'évolution des populations animales et végétale avec leur période de crises et de déprises. Malheureusement, ces données ne sont pas régulièrement enregistrées au Cameroun. On a l'impression que le Cameroun gère naïvement ses aires protégées, se contentant du maigre apport financier et laissant de côté la recherche scientifique et les mesures d'incitation pour l'aménagement es ces sites. Le suivi scientifique et l'entretien des aires protégées vont pourtant de pair et peuvent soutenir un développement harmonieux et efficient.

\section{Les recettes et fréquentations touristiques des Aires protégées}

Les données fragmentaires dont nous disposons ne concernent que les sites du Grand Nord du Cameroun. Le Cameroun avec l'ensemble des ses aires protégées ne récolte pas plus de 25milliards de FCFA (40millions de \$) pour son tourisme en 1998 alors que dans les pays comme le Kenya, le tourisme est la plus grosse contribution au PNB (419millions \$) soit 10 fois plus qu'au Cameroun. En Afrique du Sud, $70 \%$ des coûts de gestion des aires protégées proviennent des revenus touristiques générés par les parcs et réserves. En clair, ce que le Cameroun perçoit de sa demande touristique dans les aires protégées est insignifiant par rapport aux recettes d'autres pays moins bien lotis que nous comme le Kenya (1,05 millions de visiteurs) où le tourisme ne repose que sur les aires protégées et le Zimbabwe où le nombre de visiteurs a presque doublé en 4 ans passant de 375000 à 658000 entre 1988 et 1992 . Il est vrai qu'en Afrique francophone le tourisme dans les aires protégées paraît moins importante peut être pour des raisons d'aménagement, des gestion, de sécurité, de coût des transport, d'équipement, de diffusion de la publicité. En effet, pendant que des millions de touristes visitent les aires de l'Afrique Orientale et Australe, $1 / 20^{\text {ème }}$ ou $1 / 25^{\text {ème }}$ seulement visitent les sites classés d'Afrique francophone (Afrique Occidentale et Centrale); bien entendu avec le Sénégal et la Côte-d'Ivoire qui enregistrent eux aussi dans leurs parcs moins de 200000 visiteurs par an. Au Cameroun, on aurait pu se consoler d'un faible taux de fréquentation des aires protégées si ce taux était constant. Malheureusement, la fréquentation touristique des aires protégées a diminué dans tous les sens et le Cameroun n'a totalisé 10000 visiteurs dans l'ensemble de ses parcs du Nord qu'en 1982-1983 (Figuras 2a \& b). 
Figura 2a: Variation de la fréquentation touristique des parcs nationaux du Grand Nord (1964-2001).

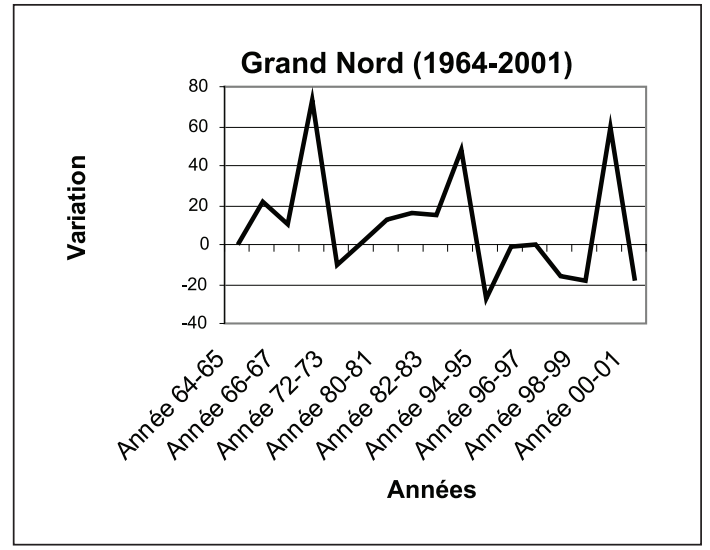

Figura 2b: Evolution de la fréquentation touristique des parcs nationaux du Grand Nord (1964-2001).

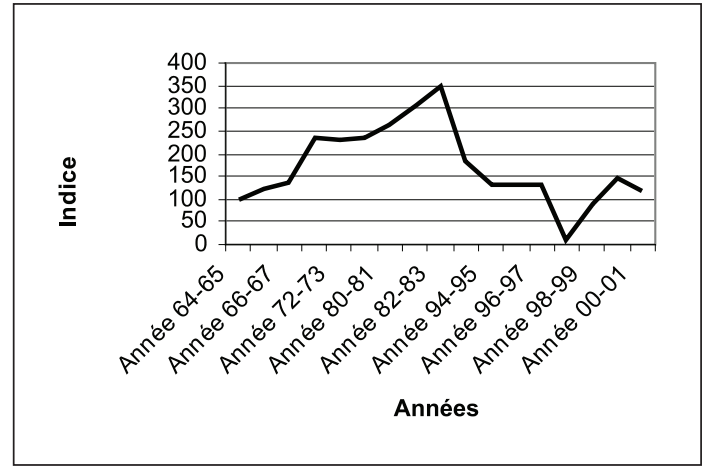

Le taux d'accroissement moyen des touristes est négatif, moins de 15\% dans l'ensemble. Des 4 sites étudiés, aucun ne montre une évolution appréciable; bien plus on a l'impression que le tourisme cynégétique de vision est mort au Cameroun; l'abattage des animaux étant privilégié provoquant conséquemment une diminution des effectifs (Figuras 3a \& b). En effet, on vend plus de permis de chasse que l'on ne paie les droits d'entrée dans les parcs nationaux. Par conséquent, au vu de tout ce qui précède, on constate que le Cameroun a multiplié les aires protégées pour la nature ou comme 
musée conservatoire fermé puisqu'il enregistre très peu de visiteurs dans ces sites sinon pas du tout. Malgré le record de l'année 1983 où les parcs de waza et de la Bénoué ont accueilli respectivement 6733 et 3000 touristes, alors que le Kenya dès 1971 accueillait 300000 touristes dans les parcs nationaux.

Figura 3a: Evolution dês effectifs dês grands mammifères du parc de Waza (19621996).

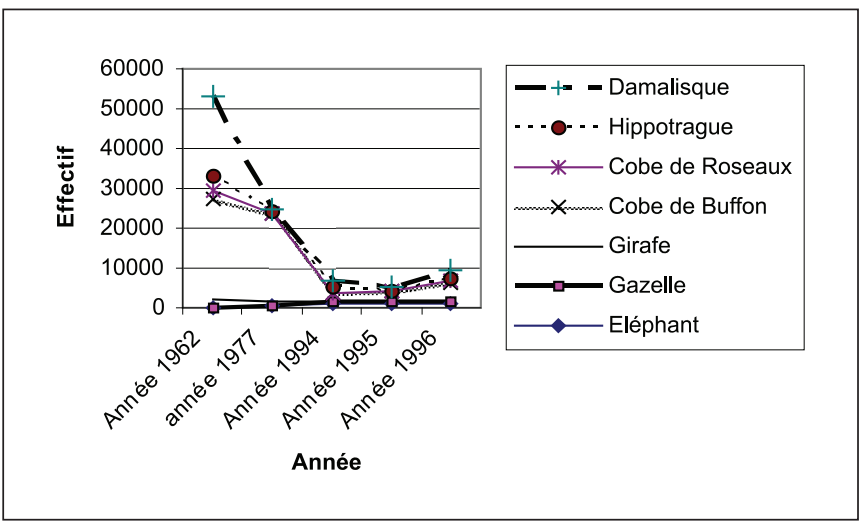

Figura 3b: Prévisions et prélèvements dês espèces animales en 11 ans.

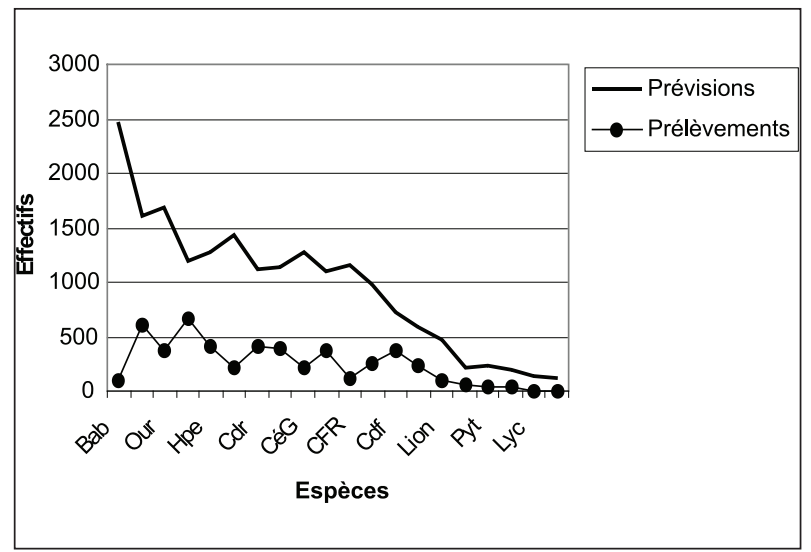

L'impression générale qui se dégage est que le Cameroun ignore complètement les conditions d'un flux. Le taux de fonction touristique de Waza 
et des autres parcs réunis est insignifiant. Il en est de même de l'indice de fréquentation touristique et de la capacité de charge; c'est ce qu'exprime le Tableau 3.

Tableau 3: Quelques indices de fréquentation touristique des parcs nationaux du Grand Nord-Cameroun.

\begin{tabular}{|l|c|c|c|c|}
\hline & $\begin{array}{c}\text { Taux de fonction } \\
\text { touristique }^{(1)}\end{array}$ & $\begin{array}{c}\text { Capacité de } \\
\text { Charge }^{(2)}\end{array}$ & $\begin{array}{c}\text { Indice } \\
\text { Foncier }^{(3)}\end{array}$ & $\begin{array}{c}\text { Indice spatial de } \\
\text { fréquentation touristique }^{(4)}\end{array}$ \\
\hline Bénoué & 0,002 & 0,001 & 13,22 & 1,6 \\
\hline Bouba Ndjida & 0,003 & 0,0002 & 6,02 & 0,03 \\
\hline Faro & 0,006 & 0,00 & 28 & - \\
\hline Kalamaloué & 0,0006 & 0,01 & 13,70 & 6 \\
\hline Waza & 0,03 & 0,02 & 13,70 & 3,63 \\
\hline
\end{tabular}

(1) Rapport nombre de touristes/nombre d'habitants.

(2) Rapport nombre de lits touristiques/nombre d'habitants x 100.

(3) Rapport patrimoine foncier touristique en ha/réserve foncière autochtone $\mathrm{x} 100$.

(4) Nombre de touristes à l'ha. par an.

Il en ressort clairement que le parc le plus fréquenté ne reflète pas sa fonction touristique minimale; c'est une situation préoccupante qui mérite une analyse approfondie quant aux causes et conséquences.

\section{Difficultés de valorisation écotouristique des aires protégées du Cameroun: causes et solutions}

\section{Causes}

Dans le contexte du $21^{\text {ème }}$ siècle, toute aire protégée doit devenir un outil de développement local, national et régional; bref, c'est un outil d'aménagement du territoire qui doit engranger des bénéfices tant sur le plan écologique et scientifique (échanges d'information sur le milieu, mise en place des systèmes de suivi écologiques et scientifiques et synergie des méthodes de prospection, e collecte et de suivi) que sur le plan social et économique (zonage du parc, maîtrise des déplacement des populations rurales, meilleure gestion des parcelles, contrôle réglementaire, création d'emplois et opportunités économiques, formation, intégration régionale et frein à l'exode rural). Dans cette optique, l'on doit oublier au Cameroun le modèle des aires protégées issues du colonialisme qui spoliaient les populations 
locales; et, en même temps, éviter d'en faire un luxe réservé aux romanciers et aux gens en mal de dépaysement ou aux chasseurs. Les sites protégés sont un instrument de gestion pour la survie des systèmes biologiques complexes dont nous faisons partie et dépendons. Pour ce faire, trois ordres de données sont utiles et requises pour la valorisation économique et sociale des aires protégées du Cameroun par l'écotourisme.

\section{Les données infrastructurelles et logistiques}

La plupart des aires protégées du Cameroun sont situées dans des zones excentriques ou difficiles d'accès. par conséquent, l'absence des services organisés et le mauvais état des routes impose des voyages longs, fatiguants et à la limité dangereux. Par ailleurs, il n'existe pas de piste d'atterrissage pour petit avion à proximité de ces parcs. Même s'il en existait, la mauvaise gestion, l'insécurité et la diminution logique du nombre de touristes étrangers a provoqué leur abandon. C'est bien le cas dans l'ensemble des parcs et réserves du Cameroun du Nord au Sud, notamment le parc du Faro, Boumba Bek, Lobéké, Korup et la réserve du Dja très isolés. De plus, le réseau routier du Cameroun ne présente que moins de $5000 \mathrm{~km}$ de routes bitumées sur les 60000 préexistantes parmi lesquels 52\% sont classées (avec 6\% bitumées et $46 \%$ en terres) et les $48 \%$ non classées. Par ailleurs, ce réseau est défectueux, inadapté et mal entretenu, voire même vétuste et inexistant par endroit. Ce défaut du trafic routier limite cruellement la fréquentation des aires protégées camerounaises et favorise le braconnage. Par exemple, les voisins nigérians ont plus accès au parc national du Faro que les camerounais; guides et gardes parcs inclus. Par conséquent, le réseau existant doit être amélioré et l'on devrait en créer d'autres. Le réseau ferroviaire quant à lui est insignifiant et ne peut guère faciliter le développement adéquat de l'écotourisme.

De surcroît, les équipements d'hébergements sont insuffisants et les plus confortables sont éloignés des sites en dehors des campements autour des parcs nationaux du Grand Nord; notamment, ceux de Waza qui ont été rénovés et améliorés. Pourtant, des projets élaborés de 1971 à 1976 et qui visaient l'augmentation de la capacité d'hébergement des aires protégées septentrionales n'ont jamais été réalisés. Ainsi, pour ne citer que le cas du Nord et de l'Extrême-Nord (les sites protégés du Sud ne connaissent aucun aménagement pour l'accueil des visiteurs), le tableau 4 suivant montre l'élan de planification proposé: 
Tableau 4: Situation des équipements d'accueil dans le Grand Nord.

\begin{tabular}{|l|c|c|c|c|c|}
\hline & $\begin{array}{c}\text { Ets. existants } \\
\text { en Nbre de lits } \\
(\mathbf{1 9 7 1 - 1 9 7 6 )}\end{array}$ & $\begin{array}{c}\text { Projet } \\
\text { d'amélio- } \\
\text { ration }\end{array}$ & $\begin{array}{c}\text { Taux d'ac- } \\
\text { croissement } \\
\text { \% }\end{array}$ & $\begin{array}{c}\text { Etablissements } \\
\text { actuels } \\
\text { d'hébergement }\end{array}$ & $\begin{array}{c}\text { Taux d'a- } \\
\text { ccroissement } \\
\%\end{array}$ \\
\hline Complexe Waza & 100 lits & 200 lits & $200 \%$ & 100 & $0 \%$ \\
\hline Complexe Kalamaloué & 04 lits & 100 lits & $2500 \%$ & - & - \\
\hline Hôtel Kousseri & - & 40 lits & - & - & - \\
\hline Hôtel Maroua & - & 160 lits & - & 424 & 287,5 \\
\hline Boukarou Mokolo & - & 50 lits & - & 44 & 88 \\
\hline Boukarou Rhumsiki & - & 80 lits & - & 52 & 65 \\
\hline Boukarou Mora & - & 80 lits & - & - & - \\
\hline Boukarou Bénoué & 68 lits & 145 lits & $213 \%$ & 84 & 123 \\
\hline Boukarou Bouba Ndjida & 32 lits & 145 lits & $453 \%$ & 32 & 0 \\
\hline Campement Faro & 04 lits & - & - & - & \\
\hline Autres hôtels & - & - & - & 384 & - \\
\hline Total & 208 lits & 1000 lits & - & 1120 lits & - \\
\hline
\end{tabular}

De plus la carte hôtelière du Cameroun corrobore cette idée et montre que cette région qui recèle pourtant des plus grands sites classés est faiblement dotée en hôtels par rapport aux grands centres comme Douala et Yaoundé. Les trois provinces qui constituent le Nord Cameroun (Ngaoundéré, Garoua, Maroua) ne totalisent pas le tiers des offres hôtelières des deux grandes métropoles de Douala et Yaoundé (Figura 4).

On constate aussi en analysant les données des fréquentations annuelles qu'il manque au Cameroun des outils de planification journalière mensuelle ou annuelle pour prévoir et relever la fréquentation des touristes.

Ainsi par exemple, il y a un vide de 5ans (entre 1990 et 1994) sur les données de la fréquentation touristique du parc de Waza. Les autres parcs manquent des données sur 15 à 27 ans (Bénoué, Kalamaloué, Bouba Ndjidah). Comment peut-on élaborer des aménagements sérieux sans données statistiques sur lesquels on peut s'appuyer pour prévoir? Les mêmes outils de planification serviraient à élaborer de nouveaux circuits allant des aires protégées aux autres pittoresques que dispose le pays. Si tout cela se mettaient en place: route, hôtel en quantité suffisante et planification, dans 10 ans, le Cameroun devrait connaître un chiffre d'arrivée d'au moins 250000 touristes pour ses aires protégées. Il tirerait ainsi le meilleur parti de son immense potentiel écologique. 


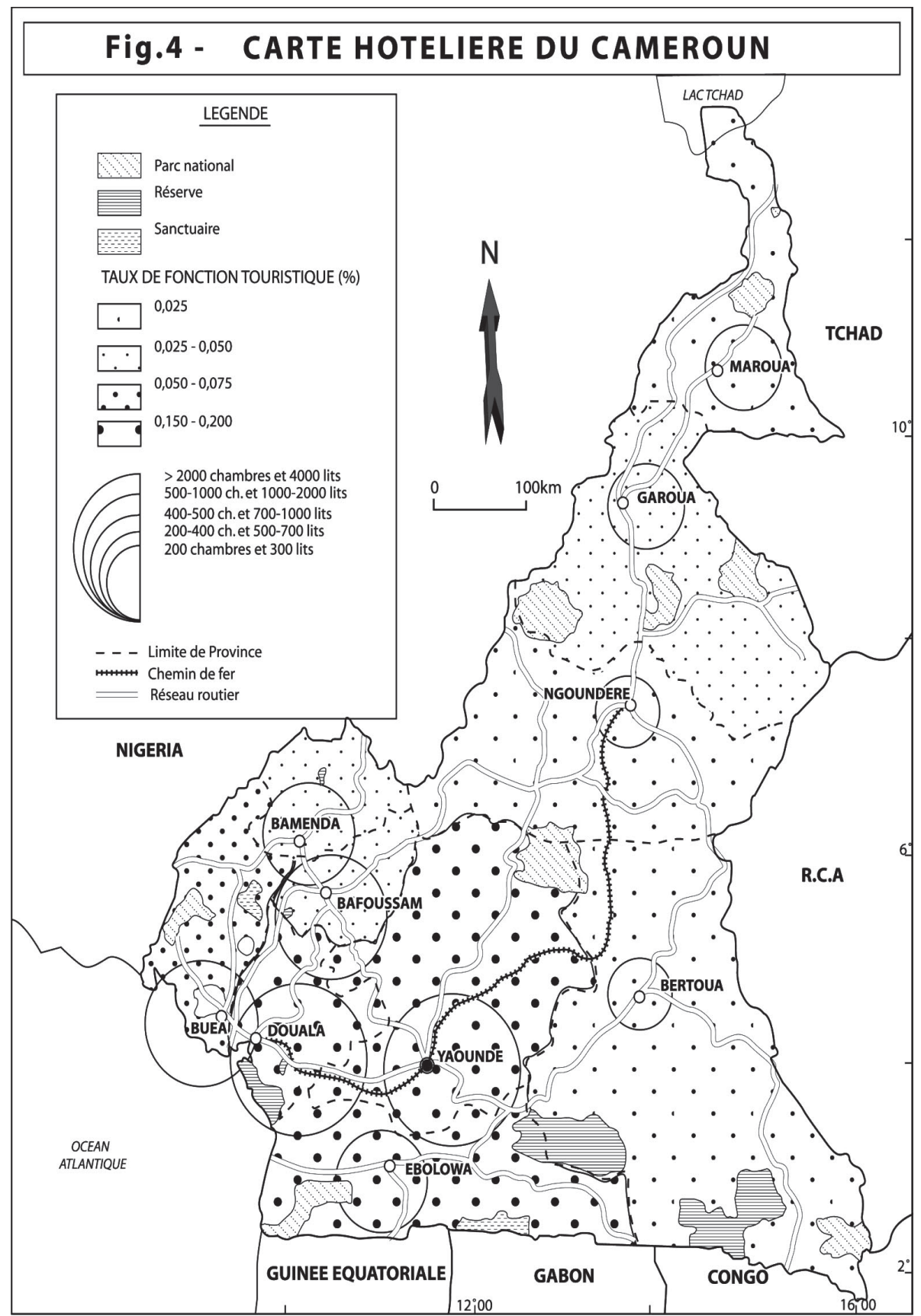


Enfin, cette valorisation est aussi rendue difficile par le très faible taux d'investissement pour la promotion touristique au MINTOUR. En effet, depuis la création de ce ministère, le budget de fonctionnement (au moins $65 \%)$ prime sur celui d'investissement (30-35\%). Même la création du Fonds Spécial De la Faune au MINFOF (Ministère des Forêts et de la Faune) ne résout que partiellement le problème. Pourtant en encourageant l'initiative privée, il est bien possible de mettre sur pied créer au Cameroun un système de campement mobile qui organiserait les circuits de safari à travers le pays; mais comment traverser les coupeurs de route!

\section{Les données scientifiques des aires protégées au Cameroun}

Depuis la mise sur pied du MAB (Man and Biosphere) par l'UNESCO, les vocations et les types d'aires protégées se sont multipliés de part le monde. Une aire protégée peut se définir comme une zone dédiée à la protection de la biodiversité et des ressources naturelle et culturelle et dont le classement relève d'un acte législatif ou réglementaire local ou/et international. Mieux, c'est une portion de terre et/ou de mer vouée spécialement à la protection et au maintien de la diversité biologique, ainsi que des ressources naturelles et culturelles associées, et gérées par des moyens efficaces, juridiques ou autres. Le Cameroun en compte 5 grands types:

- Le site du patrimoine mondial (réserve du Dja): cette dénomination caractérise une aire protégée dont les éléments biotiques ont une importance internationale exceptionnelle; ces sites sont identifiés et proposés par les Etats qui s'engagent à assumer la responsabilité de protéger ces biens dès lors que leur inscription sur la Liste du Patrimoine mondial est faite;

- Réserve de la Biosphère caractérise tout site classé dont l'objectif est de conserver la diversité et l'intégrité des communautés animales et végétales en vue d'une utilisation présente et future. Les aires protégées bénéficiant de ce statut ont une vocation de recherche, d'éducation et de formation: c'est le cas des parcs de Waza et Bénoué; les sites de réserve de la biosphère sont proposées par les pays concernés au bureau MAB et approuvés en dernier ressort par l'UNESCO;

- Le parc national est une aire protégée dans laquelle les régions naturelles et paysages exceptionnels sont protégés à des fins scientifiques, 
éducatives et récréative. Les activités d'extraction sont interdites dans ces aires qui doivent être vaste; (Parc du Mbam et Djerem, du Faro, de Korup etc.).

- La Réserve Naturelle intégrale concerne un site classé où l'on protège la nature et les processus naturels dans un état non perturbés afin de conserver des exemple du milieu dans un stade dynamique et évolutif: cas de la Réserve de Kimbi, de Mbi Crater, de Douala-Edéa et du Lac Ossa;

- Le Sanctuaire de Faune est une aire protégée dont l'objectif principal est maintenir les conditions naturelles nécessaires pour protéger des espèces ou communautés biologiques d'importance nationale si possible par l'intervention humaine: Sanctuaire de Mengame pour les Gorilles et Sanctuaire de Bayang Mbo.

Une évaluation de la faune camerounaise par le Programme National de Gestion de l'Environnement montre un potentiel immense des spécimens de faune au Cameroun. Nous les avons synthétisés dans le tableau 5 ciaprès:

Tableau 5: Situation de la biodiversité animale au Cameroun.

\begin{tabular}{|l|c|c|c|c|c|}
\hline & $\begin{array}{c}\text { Total de } \\
\text { l'Afrique } \\
\text { en \% }\end{array}$ & $\begin{array}{c}\text { Reste de } \\
\text { l'Afrique } \\
\text { en \% }\end{array}$ & $\begin{array}{c}\text { Cameroun } \\
\text { en \% }\end{array}$ & $\begin{array}{c}\text { Nbre } \\
\text { d'espèces } \\
\text { recensées }\end{array}$ & $\begin{array}{c}\text { Nbre } \\
\text { espèces } \\
\text { endémiques }\end{array}$ \\
\hline & 100 & 50 & 50 & 190 & 27 \\
\hline Mammifères & 100 & 52 & 48 & 409 & 23 \\
\hline $\begin{array}{l}\text { Papillons } \\
\text { fricains }\end{array}$ & 100 & 75 à 50 & 25 à 50 & 39 & - \\
\hline Poissons & 100 & 79 & 21 & 500 & 12 \\
\hline Oiseaux & 100 & 46 & 54 & 849 & 21 \\
\hline Reptiles & 100 & 70 à 25 & 30 à 75 & 183 & - \\
\hline
\end{tabular}

Source: tableau réalisé par les auteurs sur la base des données du PNGE

D'après ce tableau, le Cameroun nous apparaît comme un scandale écologique avec $1,6 \%$ du territoire Africain pour près de la moitié de la plupart des espèces animales. Par ailleurs en Afrique centrale et dans le Bassin du Congo, le Cameroun occupe la seconde place derrière le Congo Démocratique tant pour ce qui des forêts que de la faune. C'est l'objet du tableau 6 ci-après: 
Tableau 6: Estimation globale et comparée de la biodiversité camerounaise (végétale et animale).

\begin{tabular}{|c|c|c|c|c|c|c|}
\hline \multirow[b]{2}{*}{ Pays } & \multicolumn{6}{|c|}{ Superficies forestières en millions d'hectares } \\
\hline & $\begin{array}{l}\text { Forêt } \\
\text { dense }\end{array}$ & $\begin{array}{c}\text { Forêt } \\
\text { fragmentée }\end{array}$ & $\begin{array}{c}\text { Mosaïques } \\
\text { forêts/savanes }\end{array}$ & $\begin{array}{l}\text { Non } \\
\text { forêt }\end{array}$ & \begin{tabular}{|c|} 
Nuages et \\
eaux libres
\end{tabular} & Total \\
\hline Burundi & 0,041 & - & - & - & - & 0,317 \\
\hline Cameroun & 17,109 & 6,500 & 6,464 & 16,397 & 0,70 & 46,540 \\
\hline Centrafrique & 4,362 & 0,564 & 12,758 & 44,605 & 0,8 & 62,298 \\
\hline Congo Brazzaville & 23,325 & 1,680 & 0,913 & 7,343 & 0,389 & 34,150 \\
\hline Congo Démocratique & 112,934 & 14,813 & 30,730 & 65,224 & 3,059 & 226,760 \\
\hline Gabon & 21,188 & 1,839 & 0,295 & 2,324 & 0,120 & 26,767 \\
\hline Guinée Equatoriale & 1,678 & 0,588 & - & 0,338 & 0,200 & 2,805 \\
\hline Rwanda & 0,155 & - & 0,095 & - & - & 0,250 \\
\hline \multirow{2}{*}{ Pays } & \multicolumn{6}{|c|}{ Espèces } \\
\hline & Plantes & \multicolumn{2}{|c|}{ Plantes endémiques } & \multicolumn{2}{|c|}{ Mammifères } & Oiseaux \\
\hline Burundi & 2908 & \multicolumn{2}{|c|}{22} & \multicolumn{2}{|c|}{163} & 716 \\
\hline Cameroun & 8260 & \multicolumn{2}{|r|}{156} & \multicolumn{2}{|r|}{297} & 848 \\
\hline Centrafrique & 3602 & \multicolumn{2}{|r|}{100} & \multicolumn{2}{|r|}{209} & 668 \\
\hline Congo Brazzaville & 6500 & \multicolumn{2}{|r|}{-} & \multicolumn{2}{|r|}{200} & 500 \\
\hline Congo Démocratique & 11000 & \multicolumn{2}{|r|}{3200} & \multicolumn{2}{|r|}{415} & 1086 \\
\hline Gabon & 6551 & \multicolumn{2}{|r|}{-} & \multicolumn{2}{|c|}{320} & 617 \\
\hline Guinée Equatoriale & 3250 & \multicolumn{2}{|r|}{66} & \multicolumn{2}{|c|}{184} & 392 \\
\hline Rwanda & 4000 & \multicolumn{2}{|r|}{32} & \multicolumn{2}{|r|}{30} & 276 \\
\hline
\end{tabular}

Source: d'après Mayaux et al. (1997) et Aubé (1996).

Tout ceci n’a pour autant pas donné lieu à une véritable mise en place d'une section scientifique de suivi et de traitement des espèces animales dans les parcs nationaux du Nord Cameroun. Or l'on sait partout dans le monde que le personnel d'un parc se divise en trois sections: une section administrative, une section scientifique et technique et une section de surveillance. Au Cameroun, cette répartition n'existe pas; sinon elle est floue. En effet, le conservateur dans la plupart des cas apparaît comme une machine à tout faire, hormis ses lourdes tâches administratives et préoccupantes auquel le MINFOF tente aujourd'hui d'y remédier.

La section technico-scientifique est inexistante dans ce pays qui dispose d'une faune variée. Certes quelques études ont été réalisées ça et là sur les éléphants (population en augmentation) de Waza (Figura 5) par le biologiste Joël Okula, selon Kirda (2000) en plus des dénombrements des grands mammifères de Waza réalisés par depuis 1973 par l'Ecole de Faune de Garoua. Malgré tout, il n’existe pas sur le site même des aires protégées 
une section technique et scientifique pour l'étude et les soins aux animaux. L'école de Faune de Garoua Garde toute sa réputation, mais il faut accepter le constat de diminution de la faune (figuras 3a \& b) d'un parc à l'autre. Par ailleurs, la répartition bio et zoogéographique des animaux est peu connue. L'intérêt scientifique paraît relayée au second plan même s'il existe au Cameroun des structures scientifiques de haut niveau capable de réaliser de telles études (études d'impacts) avec le financement ou l'appui des ONG.

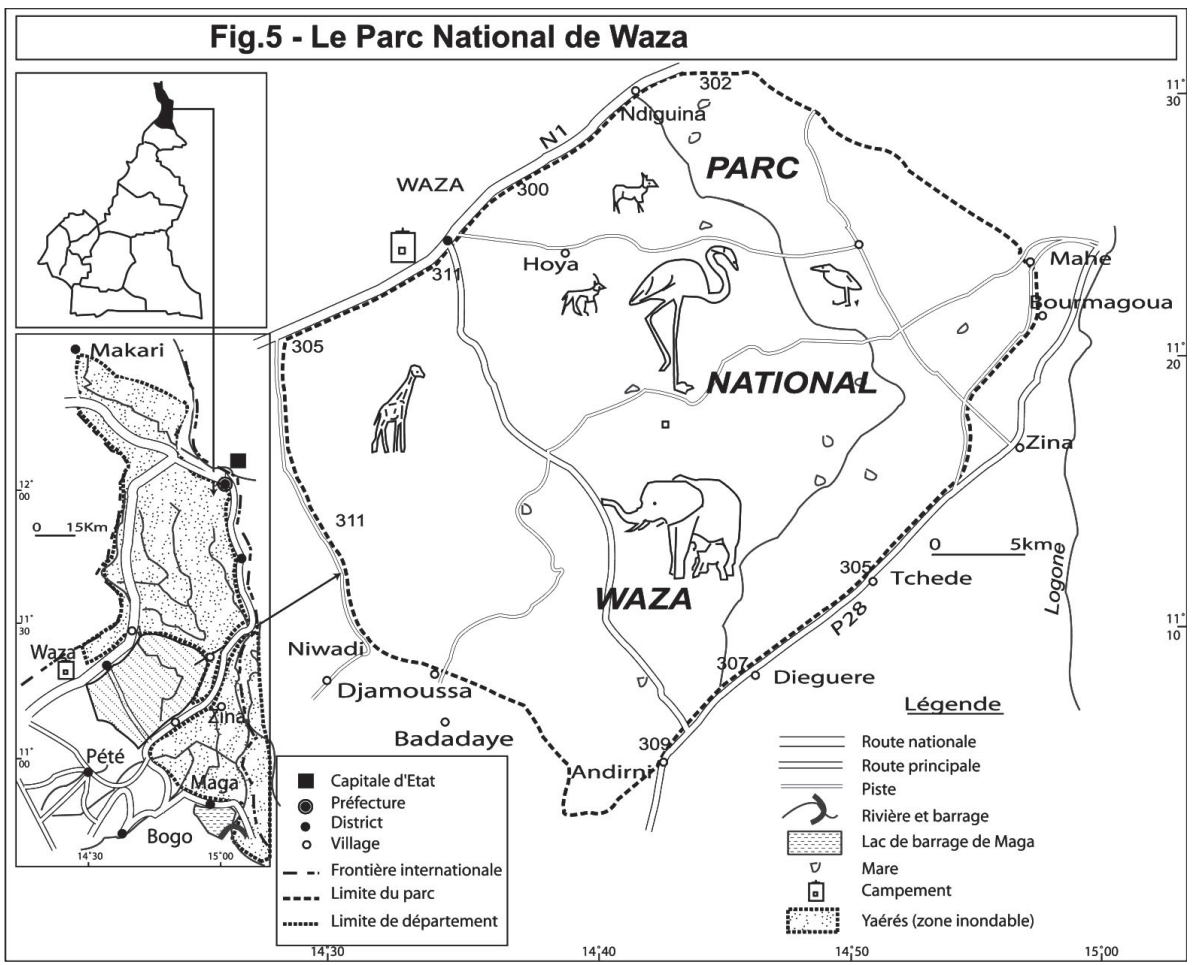

Par ailleurs, les différents ministères intervenant dans ce domaine: MINFOF, MINEP (Ministère de l'Environnement et de la Protection de la Nature), MINESUP (Ministère de l'Enseignement Supérieur), MINTOUR devraient conjuguer leurs efforts pour essayer de créer à travers le triangle national du Cameroun des observatoires des divers milieux naturels existants ou relictuels (milieux soudaniens, sahéliens, guinéens, montagnards, littoraux ou maritimes). En dehors du parc national de Korup (fig.6), aucun parc n'est véritablement zoné. Chaque parc devrait être doté d'une zone de 


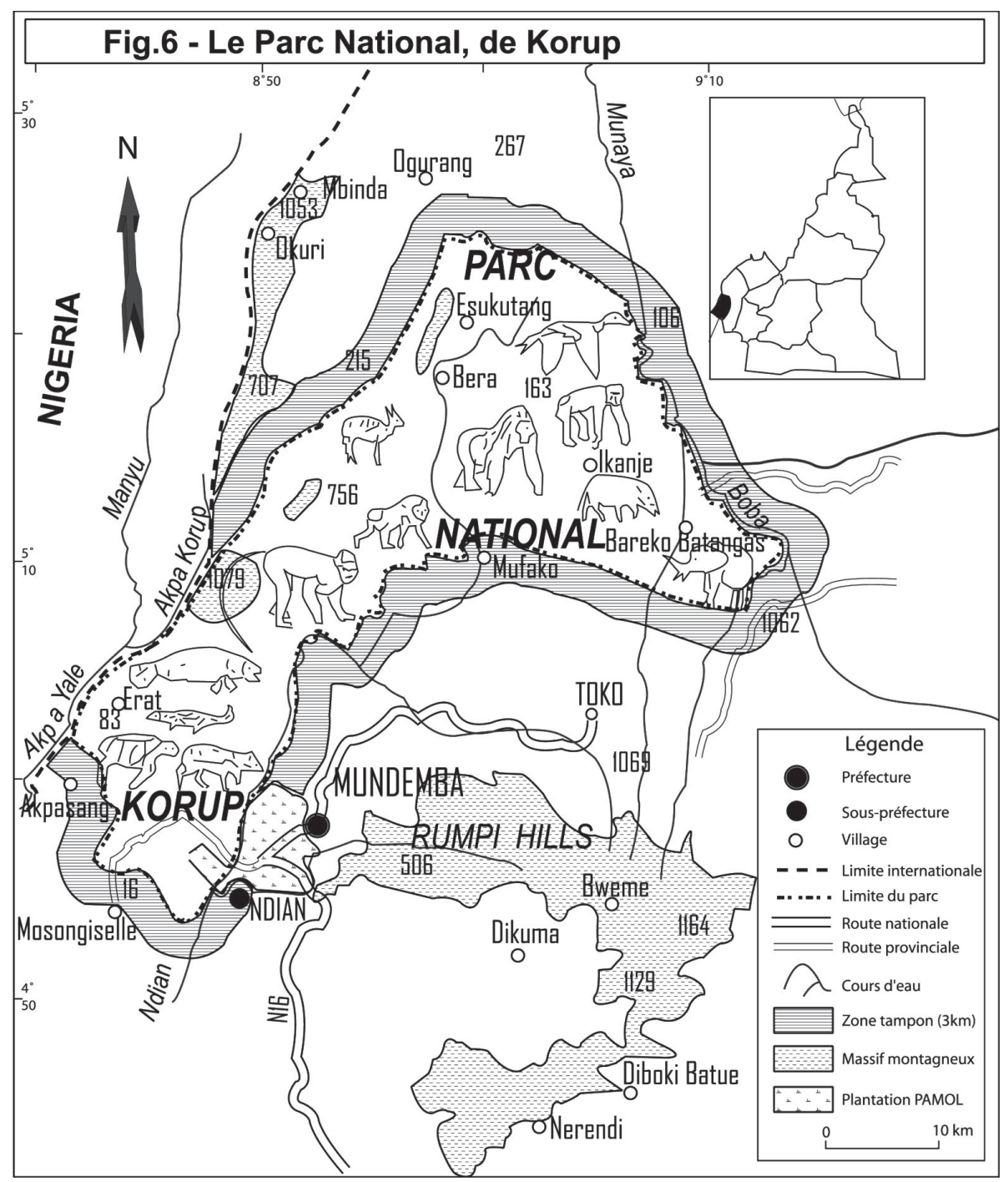

recherche ne serait-ce que par le biais des transferts de technologie conformément aux accords de la Convention de Rio sur la CDB. Les recherches doivent porter sur la dynamique de la flore et de la faune des sites protégés. Par la suite les études polliniques sont nécessaires notamment dans les milieux relictuels comme le Nord Cameroun où il existe une forte probabilité 
des traces de présence des fossiles des dinosaures dans les parcs de Bouba Ndjida et Bénoué (Figura 7). Cependant, nul ne peut ressusciter les brontosaures, toutefois, il est possible de conserver et sauvegarder les espèces actuelles menacées d'extinction comme le Rhinocéros noir dont on ne signale plus que quelques individus. La connaissance de la dynamique des populations animales et végétales peut donc autoriser ultérieurement la mise en place des stratégies de conservation, de reproduction, de protection et de survie des espèces recensées dans les différents biotopes pour une exploitation durable et un développement durable du tourisme.

Quant à la section surveillance, elle comporte beaucoup de lacunes. On compte actuellement 1 garde pour 15000ha, sinon plus; alors que normalement on devrait avoir 1 garde pour 5000ha au maximum. Sur l'ensemble des aires protégées qui existent, le Cameroun devrait former et se doter d'au moins 1200 gardes en plus du personnel administratif et scientifiques; ce qui peut porter le volume du personnel intrinsèque des aires protégées à 1500 personnes hormis les guides de tourisme. Cela nécessite une sensibilisation adéquate, des mesures incitatives en faveur de la promotion de l'écotourisme par le secteur privé, des stratégies de contrôle et une logistique appropriée. La formation étant inopérante, les risques de pauvreté s'accroissent et renforcent la pression humaine sur les ressources.

\section{Les données sociopolitiques}

Le volet social au Cameroun est marqué par le contexte économique de misère accentuée. En effet, le contexte social camerounais est précaire marqué par l'informalisation des activités. On observe une montée des exclusions qui ont engendré la marginalisation et le sous emploi (67\%), sans oublier une forte déscolarisation depuis 1990. Les risques d'aggravation des disparités régionales se font sentir et hypothèquent l'avenir. la pauvreté a gagné les ménages et le secteur informel satisfait près de 70\% de la demande de ces ménages. Le nombre de pauvres en milieu rural passe de 49\% en 1983 à 71\% en 1993 pour une population où plus de 50\% ont moins de $15 \mathrm{ans}$. Par conséquent, les secteurs les plus pauvres sont les zones rurales où sont établies ces aires protégées. Il revient donc à l'Etat d'œuvrer pour la réduction de la pauvreté afin de diminuer la pression actuelle exercée sur les ressources par le braconnage et d'autres pratiques mafieuses de ces populations appauvries. Une enquête du PNUD en 1996 (PNUD, 2000), montre bien que 


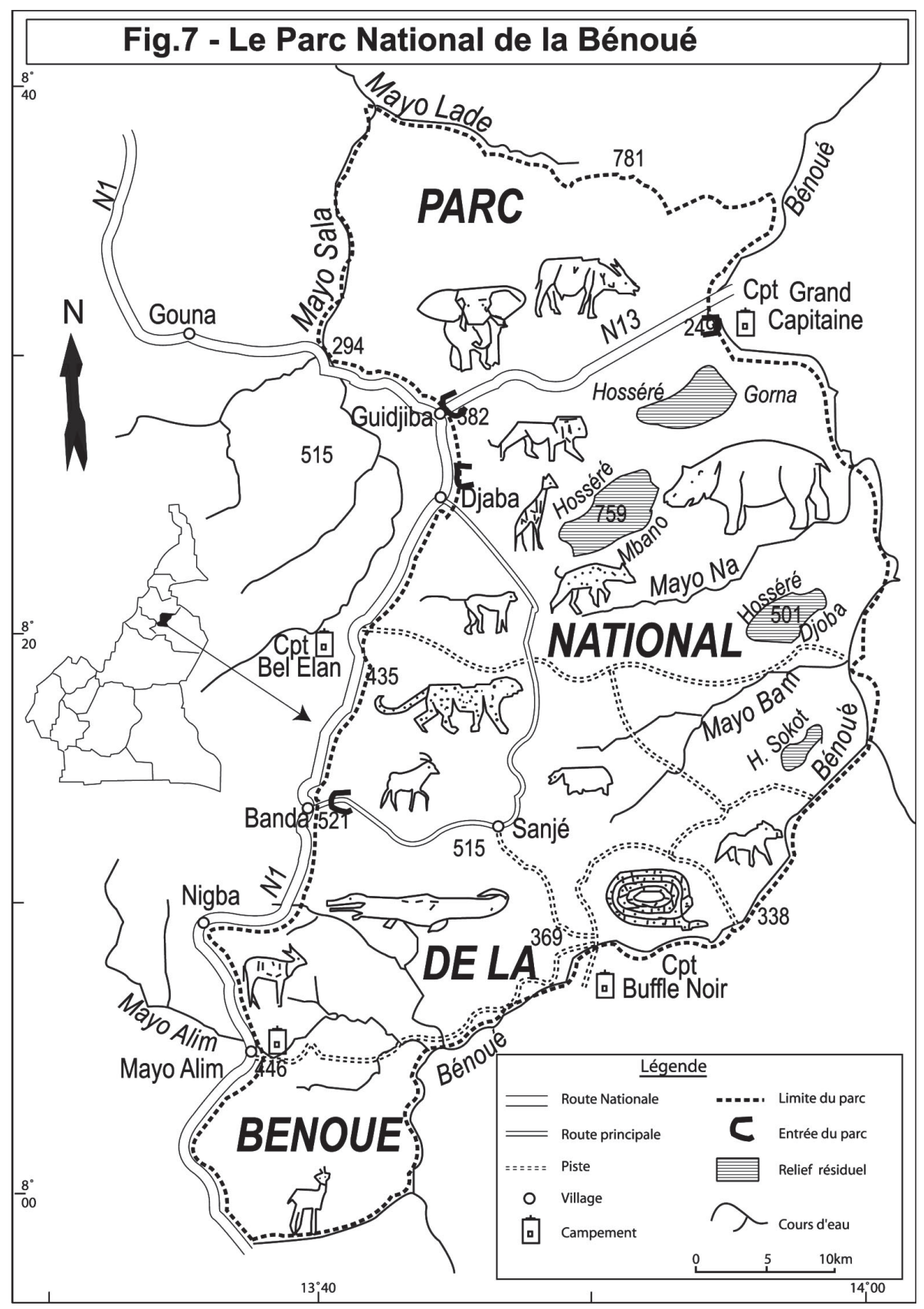


l'index de pauvreté (IP) est plus élevée dans les zones rurales forestières, de savanes ou montagnardes avec un record pour la Province de l'Extrême Nord $(\mathrm{IP}=0,49)$ suivi de la Province du Nord $(\mathrm{IP}=0,44)$, Adamaoua $(\mathrm{IP}=0,37)$ et l'Est (IP=0,30). Les trois provinces les plus pauvres abritent au moins plus de la moitié des sites protégés. Il faut donc remédier à une telle situation.

Pourtant l'augmentation nombre des aires protégées devrait être suivie par celle des gardes, ce qui pouvait diminuer la pression sur les sites sans oublier la formation des guides qui serait d'un apport substantiel. Le Tableau 7 suivant résume la situation avant et après Janvier 2000.

Tableau 7: Situation des aires protégées au Cameroun depuis 1932.

\begin{tabular}{|l|c|c|c|c|c|c|c|c|}
\hline \multicolumn{4}{|c|}{ Situation avant Janvier 1994 } & \multicolumn{4}{c|}{ Situation après Janvier 2000 } \\
\hline & Nombre & Superficie ha & $\%$ & Gardes & Nombre & Superficie ha & $\%$ & Gardes \\
\hline $\begin{array}{l}\text { Parcs } \\
\text { nationaux }\end{array}$ & 07 & 1026800 & 2,20 & & 17 & 3148937 & 6,62 & \\
\hline Réserves & 07 & 1002995 & 2,15 & & 06 & 702995 & 1,47 & \\
\hline $\begin{array}{l}\text { Zones } \\
\text { cynégétiques }\end{array}$ & 28 & 2364396 & 4,72 & & 56 & 4735250 & 9,96 & \\
\hline $\begin{array}{l}\text { Jardins } \\
\text { Zoologiques }\end{array}$ & 03 & 4,07 & 0,0008 & & 03 & 4,07 & 0,0008 & \\
\hline $\begin{array}{l}\text { Sanctuaire de } \\
\text { faune }\end{array}$ & 00 & 00 & 00 & & 03 & 246368 & 0,52 & \\
\hline Total & 46 & 4394195,07 & 9,24 & & 85 & 8833554,07 & 18,58 & \\
\hline
\end{tabular}

De plus, les gardes parcs en place sont mal payés et mal équipés; ce qui engendre sur le terrain des situations conflictuelles. En effet ceux-ci perçoivent leur salaire mensuel irrégulièrement. De plus, ils manquent de Motorola pour la communication. Ensuite, les munitions leur manquent et leur matériel de protection est vétuste; ils sont donc exposés aux animaux féroces (lions, panthère) à tout moment, car ils portent un matériel rudimentaire datant de la Première Guerre Mondiale; le MASS 36 et de surcroît sans munitions. Ces "baguettes de bois" ne sont rien à côté des fusils de calibre 12 utilisées par les braconniers. Leur maison est sans structure élémentaire: pas d'eau, pas d'électricité, pas de meuble etc. Il leur manquent jusqu'à l'essence, alors qu'ils doivent chacun couvrir au moins $150 \mathrm{~km}$ par jour dans leur secteur de surveillance. Comment peut-on leur demander avec un salaire de $80000 \mathrm{FCFA} / \mathrm{mois}$ de s'acheter l'essence pour la moto, des munitions pour leur fusils dont la dernière dotation date de 1981? Les pneus de moto coûtent 80000FCFA, de quoi engloutir leur salaire mensuel alors qu'ils dis- 
posent d'une importante charge familiale! Comment peuvent-ils réussir leur mission sans allocation d'essence alors que dans les centres villes, les administrateurs en disposent aisément? Autant de problèmes que nous avons rencontrés sur le terrain et qui fait que certains secteurs des parcs sont carrément livrés à la merci des braconniers venant du Tchad (cas du parc de Bouba Ndjida; Figura 8) ou du Nigeria (cas du parc du Faro; Figura 9). Il faut y ajouter ceux liés au phénomène des coupeurs de route qui demeure une plaie sociale dans cette région du Cameroun!

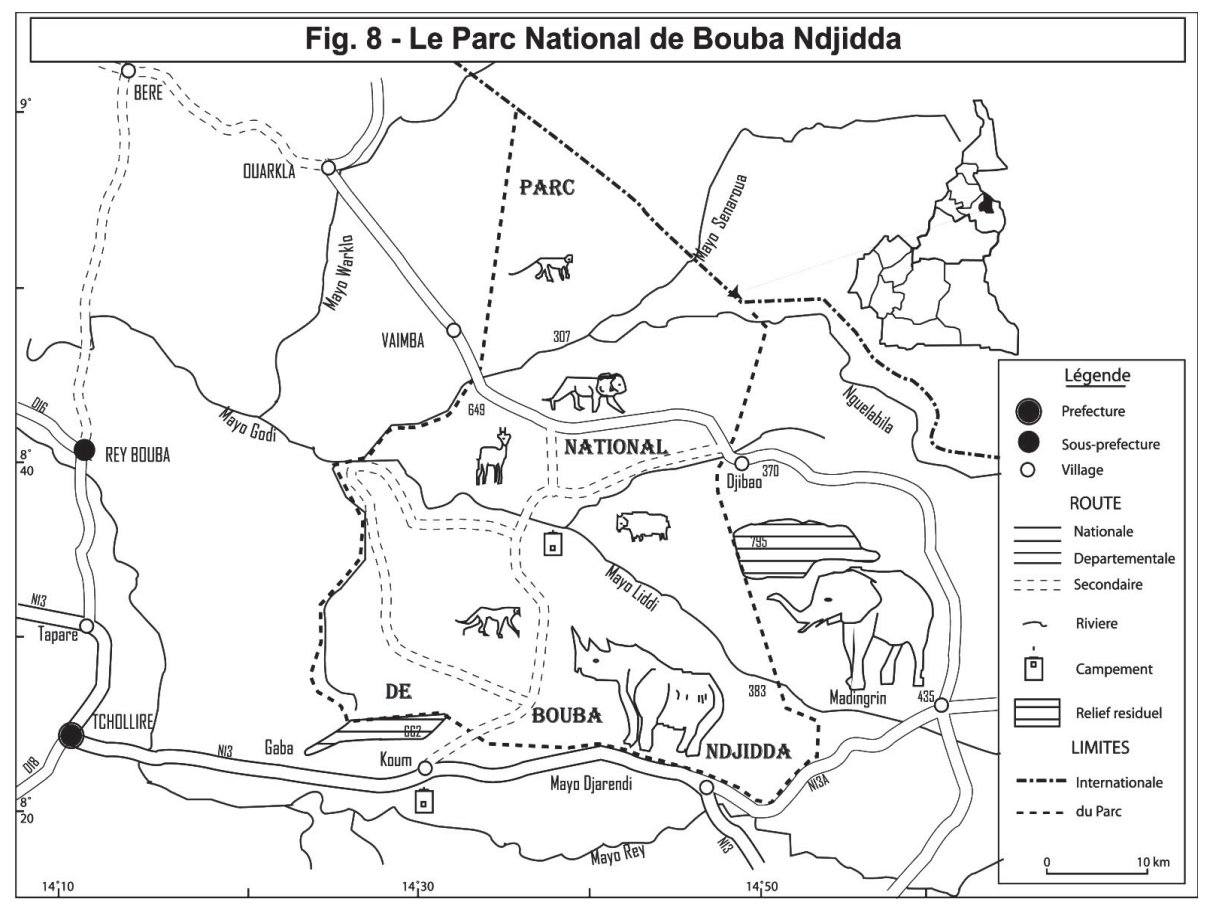

Ce problème de la pauvreté et de la misère s'accentue et crée des conflits exacerbés par la mauvaise formation ou l'absence de formation chez des gardes parcs. Ainsi dans le Département du Mayo Rey, un des gardes a eu droit à 40 jours de prison; un second a reçu une flèche empoisonnée des pasteurs en transhumance dans les ZIC vouées à la chasse. Dans de telles situations, le conservateur passe son temps à résoudre des conflits au lieu de s'occuper réellement et pleinement de la gestion du patrimoine qui lui est confié. Un Espagnol Augustin GARCIA, gérant d'une ZIC N²3 a tiré 


\section{Fig. 9 - LE PARC NATIONAL DU FARO}

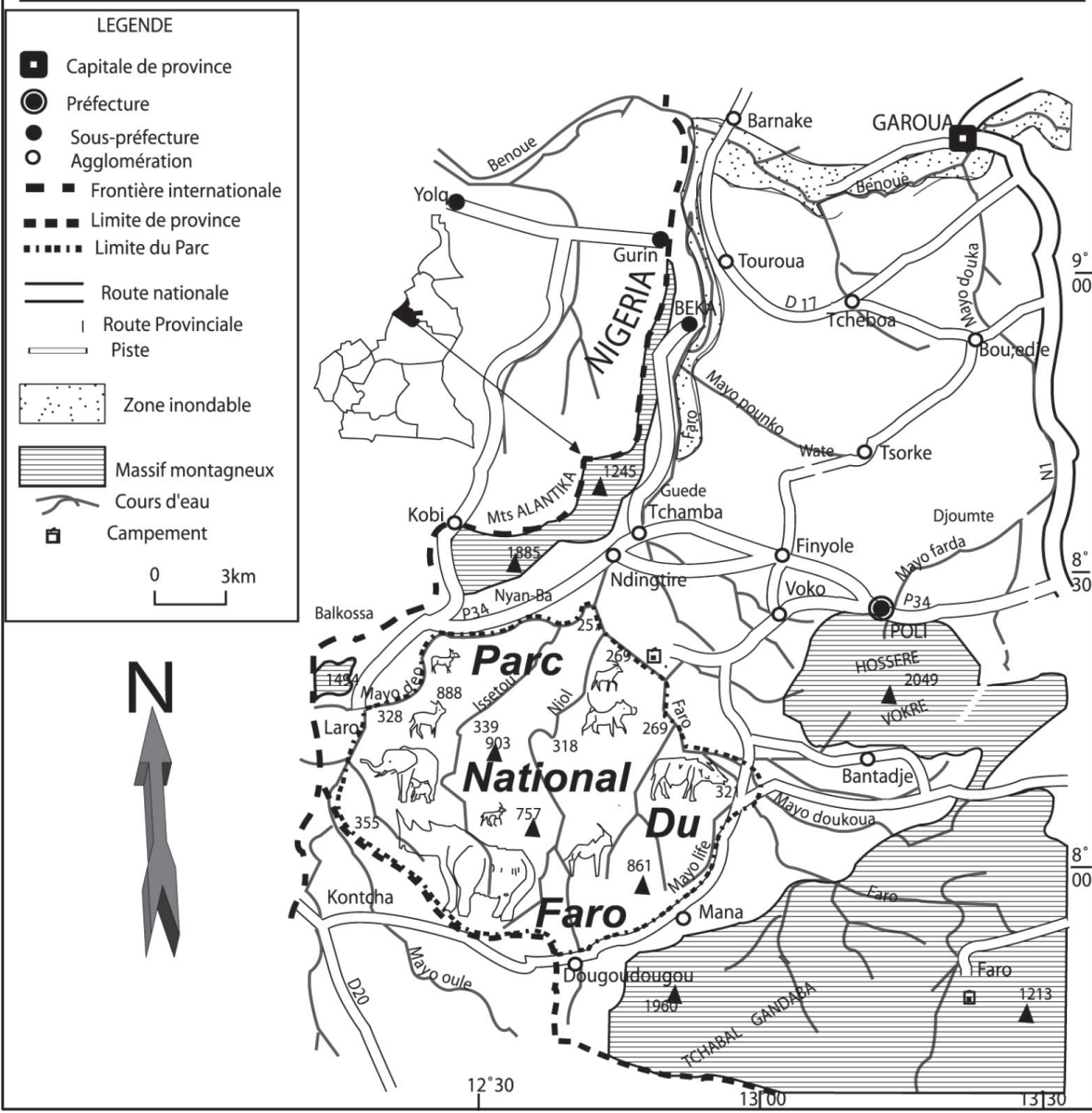

sur 13 bœufs, 9 en sont morts et il a voulu se passer des bergers qui ont porté plainte et ont eu gain de cause; ceci a obligé l'intéressé à leur verser 1800000 FCFA pour 9 bœufs morts. Or ce département est une vaste zone de transhumance qui a été réduit et cédé à des expatriés (avec 19 zones de chasse sur les 28 que compte la Province du Nord qui de surcroît porte 3 parcs de 730000 ha et une vaste zone cynégétique de plus de 2000000ha.) pour la chasse au détriment des pâturages qui ont vu leur superficie se réduire comme peau de chagrin. Il est tout de même important de faire remar- 
quer que en 1986, il existait 15 ZIC (ZIC 1-8, 9-12, 4-16; Figura 10) dans le Nord pour une superficie totale de 729390ha. En 1996, on dénombre 27 ZIC (ZIC1-5, 7-22, 23-26; Figura 10) pour une superficie de 2190048ha ce qui fait une augmentation de 200\%. En 1998, 28 ZIC (ZIC 1-5, 7-26; Figura 10) sont identifiés pour 2339160 ha soit $6,80 \%$ d'accroissement. Ces 28 ZIC ont une répartition précise: 23 sont affermées aux guides de chasse, 3 sont gérées en régies par l'Etat, 1 est attribuée au Lamido de Rey Bouba et 1 est destinée à l'expérimentation d'un game ranch.

On comprend dès lors la colère des populations qui n'ont plus de pâturages. Les terres des populations sont ainsi spoliés sans leur avis comme à l'époque coloniale et ce peuple de pasteurs ne réclame que la rétrocession de ces zones de pâturages: notamment la réduction de la ZIC N ${ }^{\circ} 23$ et la rétrocession de la zone du Mayo Boddo annexée à la ZIC N 25 en plus de la suppression pure et simple de la ZIC $N^{\circ} 10$ avec libération des colons pour la circulation des animaux en transhumance (Figura 10). De plus, les recettes tirées de l'exploitation des ces ZIC sont minables: 1166375\$US en 15 ans d'abattage (soit $77758 \$ /$ an) et 19581 \$ pour le tourisme de vision.

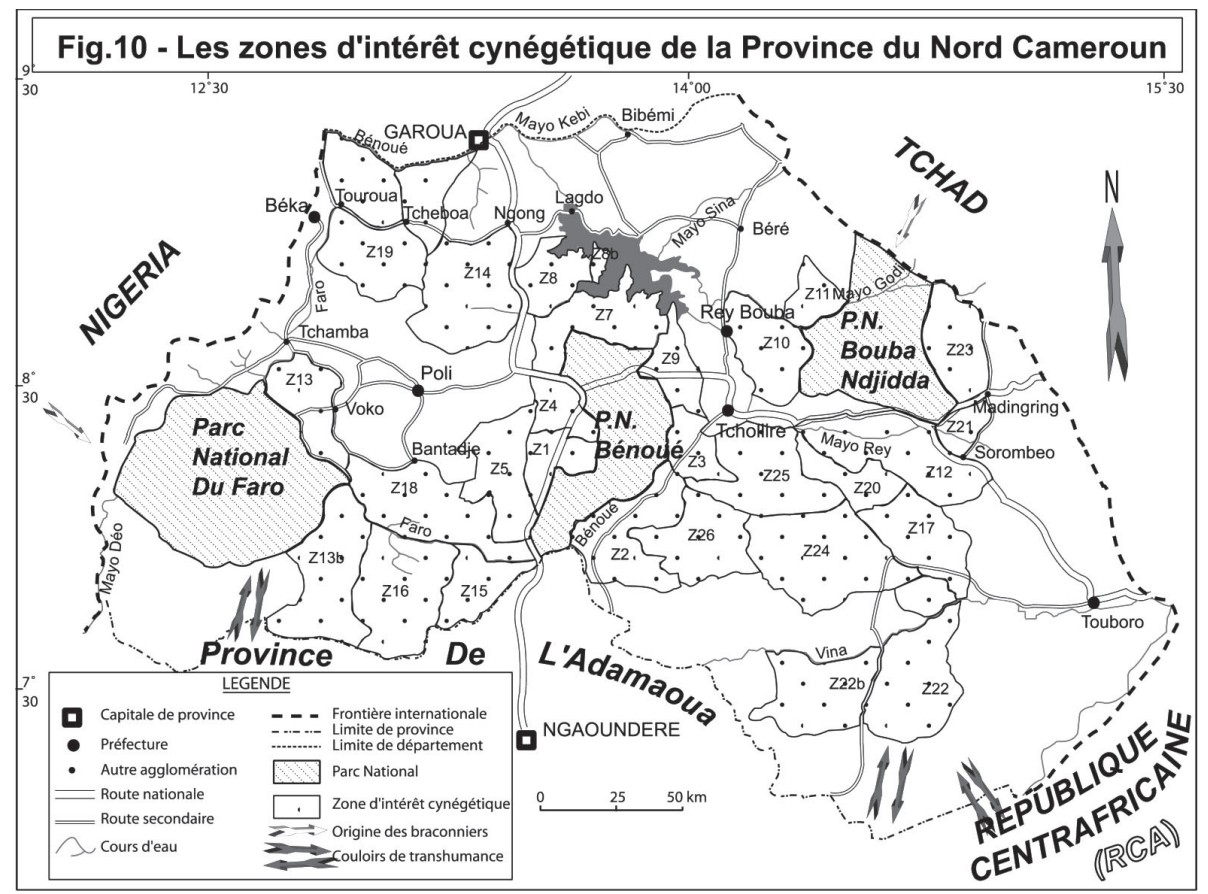


Entre 2000 et 2003, le Cameroun a créé 28 autres zones d'intérêt cynégétique (Figura 11) dans le sud forestier. Cette création vise quel objectif lorsque l'on sait que les zones d'intérêt cynégétique sont gérées par les étrangers (Expatriés) De plus ces ZIC dans la partie méridionale du Cameroun sont bordés ou intégrés aux unités forestières d'aménagement (UFA).Ceci constitue une autre raison de conflit sociopolitique! En effet, comment les politiques pourraient-elles protéger des secteurs classés en y multipliant des domaines de chasse et d'exploitation forestière. Les UFA et les ZIC ne peuvent pas favoriser les déplacements d'animaux dans leur domaine naturel et coupe même les corridors prévus pour leur épanouissement. Dans le même temps, l'exploitation du bois réduit la biodiversité en qualité et en quantité en termes d'espèce végétale et animale. C'est une preuve du manque d'unité scientifique de suivi dans des aires protégées du Cameroun.

Le problème de la pauvreté s'ajoute à celui de la formation et à l'analphabétisme, en plus des retards de salaire. L'accentuation de la misère dans une zone sèche pousse les grades parcs et les populations en place à une situation de révolte sociale dont le phénomène des coupeurs de route en est une illustration parfaite. Les grades parcs participent eux mêmes au braconnage de 2 façons:

- Soit par un silence complice;

- Soit par une déclaration inexacte et fausse du nombre d'animaux pour permettre au MINFOF, Ministère de tutelle de programmer et planifier les tirs ou les plans d'abattage des animaux. Par exemple, s'il y 60 éléphants et que le plan de tir en prévoit 10 (pour ne pas descendre au dessous du seuil de 50) par an, le garde parc va déclarer 70 pour donner lieu à 20 abattages; ce qui ramène la faune des éléphants à 40 au lieu de 50. C'est ce qui justifie en partie la diminution du nombre des animaux dans les parcs et autres aires protégées. Les plans de tir se basant sur de simples allégations des guides de chasse, il est normal que les effectifs des populations animales diminuent parce que les gardes perçoivent certaines espèces comme concurrentiels aux espèces gibiers. On comprend dès lors la mission dévolue aux scientifiques à travers la formation dispensée par l'Ecole de Faune de Garoua qui assure certes une formation de qualité. Mais celle-ci demeure encore insuffisante, dispersée et moins spécialisée. En effet une telle formation devrait se poursuivre sur la pointe de la technologie pour donner lieu à des docteurs spécialistes du système de reproduction et données sociologiques des populations animales des parcs. Par ailleurs la formation des agents de 


\section{Fig.11 - Les Zones d'intérêt cynégétique du Sud Cameroun}

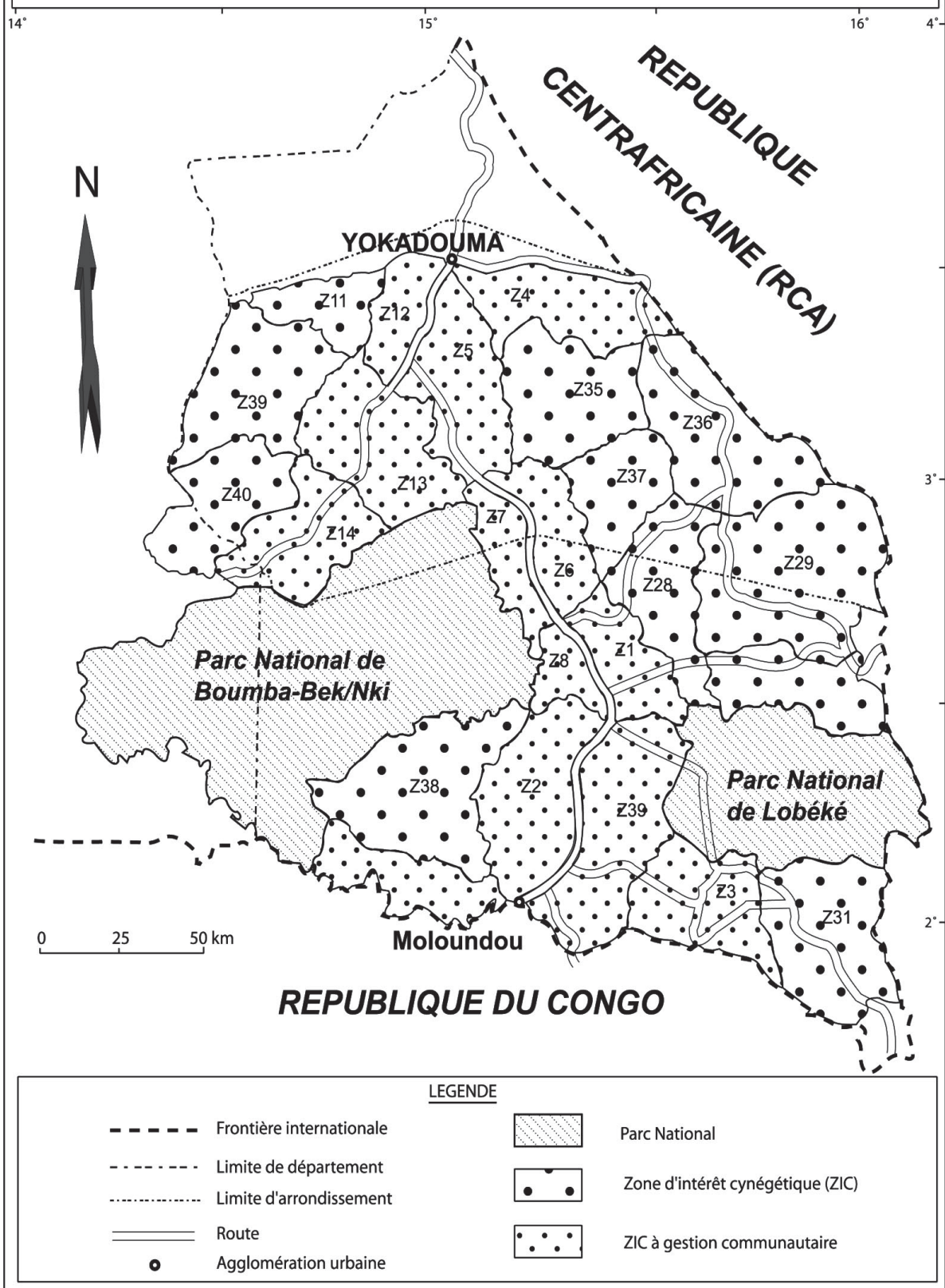


surveillance rencontrée dans les basses couches est un casse tête chinois; ceux-ci remplissant leur mission plus par ignorance et zèle que par empirisme et connaissance suffisante; dans ce sens, leur rôle apparaît comme une forme de sanction pour la faune sauvage.

Pourtant l'écotourisme (le tourisme) apparaît comme un levier important de lutte contre la pauvreté parce qu'il permet le développement des petits métiers d'artisanat étroitement liés à la culture locale et emploie une forte proportion du genre féminin. Il faut y ajouter le développement des produits complémentaires telles que: classes de danse, compte culturels, cours de cuisine locale, visite des écoles, visite des villages artisanal, la découverte des plantes médicinales locales par les soins d'un guide, le partage convivial des mets locaux etc.

Ce tableau sombre montre simplement que la gestion participative n'est pas la chose la mieux partagée au Cameroun. En effet l'implication des populations dans la gestion du patrimoine de leur ressort territorial est un échec dans cette région du Nord Cameroun parce que l'exercice du droit d'usage n'est pas bien défini. En effet comment protéger et conserver les animaux dans un parc en créant la misère et en affamant les populations alentours? Quelle est la meilleure optique de conservation et de dialogue? La destruction de l'autorité locale traditionnelle n'accroît-elle pas des conflits sociaux et la pression sociale sur les ressources?

Si les populations riveraines ne sont pas considérées comme actrices et partenaires des ZIC avec des devoirs et des droits; la conservation de leurs ressources est-elle encore possible? On sait pourtant qu'une meilleure connaissance et gestion des aires protégées nécessite dans l'optique de la lutte contre le braconnage et de la stabilisation des ressources une gestion participative par l'implication (et non l'imposition) des populations riveraines dans l'organisation et la gestion de l'espace-parc, ainsi que sa valorisation à trois échelles:

1. Au niveau des débats: le Cameroun a une pléthore de tribus répartis sur l'ensemble du territoire National. Les parcs et leur utilité seront appréhendés différemment selon les régions et les groupes ethniques. Ainsi, que pense un riverain de Mundemba du Parc National Korup? Que pense l'homme de Campo ou le pygmée de Bipindi de la conservation et de la protection de son environnement? Qu'entendent ces populations par créer, classer, préserver, protéger? comment perçoivent et conçoivent-ils à leur niveau le développement durable? Quel peut être alors leur apport et leur 
effort de valorisation? Toutes ces questions et bien d'autres sont des préliminaires ou des préalables à toutes prise de décision objective et efficace.

2. Au niveau des prises de décision: Après la conduite des débats dans toutes les couches de la société, les décisions prises ou à prendre doivent tenir compte des avis et réserves émis par les populations riveraines des aires protégées. Pour ce faire, il est nécessaire de créer des cadres de discussions avec les villageois pour des rencontres périodiques dont la finalité est de situer, évaluer, et prendre en compte la façon des riverains décrypte, déchiffre leur milieu et considèrent leur patrimoine. Car il faut le reconnaître, sans vergogne, ces milieux précaires aujourd'hui étaient mieux conservés jadis, au moment où la "civilisation moderne" qualifiée de civilisation vampire de l'environnement, n'avait pas atteint ces contrées.

3. Au niveau de la surveillance effective du parc, 2 étapes sont nécessaires. Il faut dans un premier temps donner des rudiments scientifiques des espèces et de leur importance écologique avec identification de ces espèces dans la langue locale en même temps qu'une éducation environnementale de base. Dans un second temps la formation des guides, gardes parcs et gardes chasses en nombre suffisants est incontournables. Ion en compte actuellement 15 au Faro, 22 à la Bénoué et 18 à Bouba Ndjida et 25 à Waza (pour les gardes parcs); jusque-là, ce chiffre est insuffisant et il faut le ramener à un garde pour 5000ha car un pour 15000 est tout simplement ridicule, et chacun de ces parcs doit en compter au moins 50. Ces vigiles pour mieux mener leur tache doivent compter parmi eux des villageois formées et équipés pour la circonstance. Ces derniers pourront très bien participer et contribuer aux travaux d'aménagement de construction et restauration des infrastructures et devenir de bons guides pour des visiteurs avides qui n'en demandent pas plus pour leur satisfaction. L'exemple du parc de Waza ou l'on compte 95 gardes recrutés (1 garde pour 1700ha) parmi les locaux (Dans la cas de la Bénoué, les grades parcs viennent de loin) peut devenir un modèle qui peut permettre la cessation de l'appropriation administrative ou de la confiscation des ressources en même temps qu'une bonne maîtrise et partant un meilleur suivi et une meilleure conservation des ressources.

Au plan politique la multiplicité des institutions de gestion qui interviennent dans la chose touristique sans coordination; ensuite, ces institutions ont des visions et intérêts divergents: MINFOF pour la Gestion 
des Aires protégées, MINTOUR pour la gestion des campements des sites protégées, MINATD pour l'administration territoriale, MINEPAT pour les investissements publics et l'aménagement du territoire, MINAGRI pour l'Agriculture, MINEPIA pour élevage et pêche etc. Une telle situation engendre des conflits de compétence sans oublier que les travaux d'entretien des pistes sont du domaine du Ministère des Travaux Publics et des Transports.

Par ailleurs, la promotion du tourisme de découverte est très faible sinon inexistante au Cameroun; en effet, il existe peu d'affiche, peu d'ouvrages et de dépliants, pas de tee shirts et divers gadgets souvenirs sans oublier franchement l'incompétence des responsables en charge des dossiers. La délimitation des zones tampon entourant les aires protégées ne correspondent pas à la définition requise pour les aires protégées et rend difficile le zonage. Par exemple le passage de la Route Nationale $N^{\circ} 13$ (Figura 7) en plein parc de la Bénoué et au Sud du parc de Bouba Ndjida favorise le braconnage. Enfin les populations locales n'y trouvent aucunement pas leur intérêt parce qu'il n'existe aucune promotion de la culture locale alors que cette zone de la Bénoué et de Bouba Ndjida pratiquent un élevage diversifié et pratique l'artisanat des peaux sans oublier l'élevage équin (235 têtes) et asins (583 têtes) qui sont utile pour la fantasia.

Pour ce qui est du parc de Waza, la mise en service du barrage réservoir de Maga (voir carton Figura 5) en vue de faciliter la riziculture avait porté un coup dur aux migrations saisonnières des animaux dans le parc. Heureusement après des études poussées, une solution de re-inondation du Logone a été trouvée et exécutée, ce qui a permis la restauration du parc.

\section{Solutions}

Dans notre texte nous avons ébauché quelques solutions, nous voudrions mettre plus d'accent sur certains aspects pour aider le Cameroun à sortir de ces difficultés. Il faut déjà relever que d'après l'IUCN (1990), le réseau d'aires naturelles protégées de chaque pays doit être conçu en fonction des ressources et des besoins de conservations de ces ressources dans l’intérêt du développement économique durable.

- Le pays doit mettre en place une politique d'aménagement claire et d'encouragement du tourisme et des initiatives en faveur de celui-ci! 
- Mettre en place une banque de données fiables et un bon système d'information de la base à l'intérieure et à l'extérieur par le biais des représentations diplomatiques camerounaises à l'étranger ainsi que celui des agences de voyage et de compagnies aériennes.

- L'information doit être donnée au touriste au départ et non à l'arrivée, notamment des informations techniques.

- La chaleur de l'accueil à procurer aux touristes ne peut venir que de la formation d'un personnel compétent pour l'hôtellerie et des guides pour visiteurs

- Le Cameroun doit accroître sa capacité d'hébergement sur les sites protégés et multiplier les moyens de transports et les infrastructures aériennes et routières garantissant aux clients un séjour agréable! accroître le réseau routier marche de pair avec la diversification des attractions touristiques dans les sites du pays et étendre celle-ci dans la sous région comme l'a si bien réussi l'Afrique Australe. Ainsi un touriste de passage au Cameroun devrait visiter après un ou deux pays du Bassin du Congo en l'occurrence, le Tchad, le Gabon la RCA etc. cela favorise la coopération sous régionale et peut permettre d'organiser une meilleure lutte contre le braconnage.

- Le Cameroun doit clarifier les objectifs de la privatisation et confier celle-ci prioritairement aux Nationaux plutôt qu'aux expatriés comme c'est le cas actuellement pour le cas parcs de Bouba Ndjida et Bénoué. En effet, une bonne gestion par un homme du terroir est une contribution importante à la prospérité nationale. L'Etat se doit de donner priorité au secteur privé tout en étant moins regardant sur les taxes. En effet au Kenya, on signale plus de 200 entreprises privées opérant dans le domaine du tourisme. Au Cameroun il n'en est pas question pourtant c'était l'une des orientations générale de la Convention de Lomé en mai 1990.

- Tous les mots existent pour qualifier le braconnage: crime, vol etc., mais, il n'existe pas de mot pour qualifier la misère. Tous les moyens existent pour combattre le braconnage (embuscade, patrouille, sensibilisation...); mais il n'existe pas de moyen pour combattre la misère; mieux, il existe un seul moyen: sortir les populations de la misère par un dialogue franc et la prise en compte de leurs problèmes en procédant par des négociations face aux situations conflictuelles. Cette solution négociée pourrait permettre de combattre efficacement l'insécurité ambiante dans cette Province avec les coupeurs de route. De toutes les façons, associer les populations riveraines 
des aires protégées à la gestion et au contrôle de celles-ci est le gage même de leur pérennité et stabilité et requiert de la part du Gouvernement des subventions comme moyens d'engouement et d'incitation des populations à la prise de conscience des richesses de leur patrimoine foncier. En effet, prendre les sites protégés comme richesse et patrimoine (dont la pérennité dépend de leur mode de gestion) nous semble incontournable pour les ruraux et les sites de plus de 100000ha devraient disposer d'au moins 100 gardes parcs. Les populations riveraines dépendantes directement des ressources naturelles ne demandent qu'à être sensibilisées, éduquées à la conservation et gestion raisonnée de leur ressources. Dans ce sens, l'exploitation touristique des parcs nationaux (visites guidées à pied, à cheval, en voiture, en pirogue etc.) en sera davantage renforcée et mieux valorisée. Une gestion à l'africaine peut être également prospectée comme c'est le cas au Kenya avec les campements mobiles ou temporaires à l'air libre avec des vigiles. Dans tous les cas, les aménagements doivent être adaptés aux conditions locales de ce type de clientèle.

- Le Cameroun qui disposait en 1960 de 20000000ha de forêt dense avec 300 essences exploitables sur les 12000 existantes a amorcé en 1994 un plan de zonage de sa forêt. Cette délimitation qui matérialise la volonté de l'Etat devrait se poursuivre de façon effective dans les aires protégées où l'on doit véritablement retrouver une zone interne, une zone touristique, une zone scientifique et une zone tampon (pour les activités agricoles, car les pratiques agricoles ou agro-pastorales autour du parc peuvent provoquer la déprise de celui-ci) comme cela se fait partout ailleurs dans ces sites avec dénombrement éventuel de la diversité biologique. La négligence à l'endroit de ces règles peut induire des changements de milieu selon la dynamique de la biodiversité animale et/ou végétale. Pour les résorber, les sites protégés peuvent servir de milieu d'expérimentation et de recherche pour l'aménagement et la transplantation (voire reproduction et protection) des espèces menacées. Tout ceci requiert des mesures environnementales sérieuses, courageuses et coûteuses (appuyées sur des données scientifiques fiables), mais, indispensables pour assurer un développement durable par l'écotourisme; et, réalisables si les populations locales en sont mieux informés et bien impliquées! Pour ce qui est du côté scientifique et de la recherche, les étudiants chercheurs des différentes universités du pays pourraient bénéficier des bourses pour réaliser des travaux de terrain en compagnie des gardes parcs. Dans ce sens des études d'impacts bien menées par eux 
permettraient au MINFOF (Ministère de tutelle) de localiser des secteurs difficiles et de monter des scénarios d'étude intégrées allant du botaniste au zoogéographe etc. En effet, il est impossible que sur le terrain, les agents du MINFOF soient en même temps juge et partie. Ces études d'impacts ont l'avantage de favoriser la planification qui s'accompagne du suivi - évaluation des différents projets amorcés.

- Entre autres propositions, il est possible de créer des haies autour du par cet financer leur entretien par la population rurale. L'on peut également entretenir les zones intermédiaires et les alentours des sites protégés en luttant contre la surexploitation forestière par des mesures d'incitation. Dans ce cas, il est urgent de déterminer les rythmes et diamètre de coupe et de ramassage du bois.

- Il apparaît indispensable pour les parcs du Cameroun de mettre en place des salines et miradors pour favoriser la vision des animaux en même temps de créer des observatoires de la dynamique des espèces afin de prévenir les modalités des phénomènes futurs.

- Enfin, cet ensemble de travaux demande une cartographie minutieuse et précise et la mise en place de systèmes d'informations géographiques (SIG, inexistants actuellement) sur chaque aire protégée. Ces SIG seront utiles pour déterminer les secteurs d'intervention, localiser les interfaces (écotopes, écotones), mesurer ou quantifier les transformations, leur rythme et leur vitesse pour infléchir si possible les évolutions. Une cartographie précise de chaque parc ou site en découlera appuyée des observations de photographies aériennes et complétée par l'information satellitaire récente. Ces méthodes utiles et si précieuses permettent d'observer, d'apprécier les rythmes de dégradation depuis la création des parcs et réserves ne serait-ce que qu'au niveau des hydrômes, phytômes et par conséquent des zootômes. Cette cartographie multi scalaire débouchera sur la confection publicitaire des dépliants donnant des informations abrgégées et utiles sur les sites avec leur localisation et leur richesse pour le plaisir des touristes. Certes le GLOBAL FOREST WATCH (de concert avec le MINEF et Le WORLD RESOURCES INSTITUTE) a amorcé un important volet cartographie en mettant à jour l'Atlas Forestier Interactif du Cameroun, Mais en réalité, beaucoup reste encore à faire, car des parcs comme celui de Mbam et Djérem (Figura 12) créé en 2000 à cause du déclassement de l'ancien parc national de Pangar Djérem traversé par le Pipeline Tchad -Cameroun, n'existe que de nom (pas de route, pas de piste etc.). 


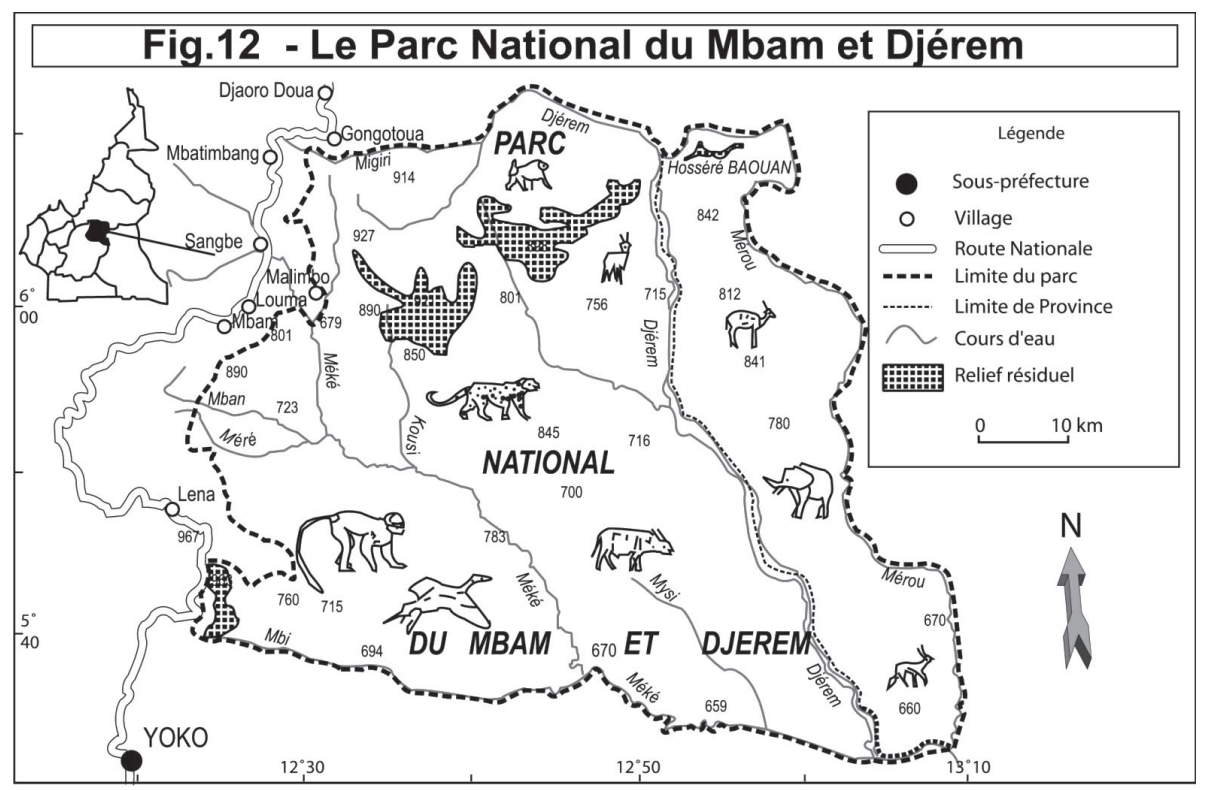

\section{Conclusion}

Le tourisme certes, n'est pas une panacée ni une maladie honteuse (comme l'a si bien signalé Pierre Favre); mais c'est tout de même un secteur de l'économie en pleine croissance sur le marché international. C'est ce qui nécessite l'attention qu'on doit lui accorder. Cependant, au Cameroun il est à l'état de balbutiement et requiert la mise en place de moyens efficaces pour le rendre compétitif dans la sous région. La présentation des difficultés camerounaises en matière d'écotourisme vise également la mise en place des mesures adéquates pour sauver ce patrimoine naturel irremplaçable que sont les aires protégées. En effet, l'idée de patrimoine devrait guider toute prise de décision tant par l'administration que par les populations riveraines côtoyant les richesses. Il s'agit bel et bien de protéger ce patrimoine en faisant aussi appel à la culture locale riche dans ses symboles pour la développer et diversifier sa promotion touristique. Dans ce sens, l'inconscience pourrait disparaître et les retards accumulés par le Cameroun dans ce secteur doivent être étudier, provoquer des remises en question et permettre d'affiner des stratégies et modes de gestion appropriées. Un partenariat réel devrait s'établir entre le Gouvernement, le secteur privé et la société civile 
voire les populations locales en vue du développement de l'écotourisme et de la réduction de la pauvreté. Les ONG en accord avec les populations locales et le Gouvernement devraient déterminer les interprètes des parties avant de s'engouffrer dans la gestion et ce n'est qu'à ce titre que le Cameroun évitera d'hypothéquer l'avenir de son immense potentiel patrimonial et touristique.

Remerciements: Nos remerciements vont à Mr KOULAGNA KOUTOU Denis, Directeur de la Coopération au MINFOF qui par autorisation spéciale nous a permis de consulter les archives existantes sur les parcs nationaux; et, à Mr MBOHOU Souleymanou, Directeur des Normes et Procédures au MINTOUR, qui a bien voulu lire le manuscrit et pour ses précieux conseils dans la réalisation de ce travail.

\section{Bibliographie}

JEUNE, Afrique. Atlas de l'Afrique. Edition du Jaguar, Paris, 2000.

BEKIMA, Louis Victor. Evaluation du mode de gestion de l'Ecotourisme dans les Provinces du Nord-ouest et du Sud-Ouest Cameroun. Mémoire DESS, Université de Yaoundé I, Yaoundé, 2000.

BINCU, Yvette. Potentialités biophysiques et perspectives d'aménagement écotouristique dans le massif du Manengouba. Mémoire de Maîtrise, Université de Yaoundé I, Yaoundé, 2000.

CANOPEE N 13. L'Afrique Centrale et le tourisme de nature. ECOFAC, Libreville, 1999.

CANOPEE N ${ }^{\circ} 20$. A quoi servent les aires protégées? ECOFAC, Libreville, 2001.

CANOPEE N² 21. Y a-t-il surexploitation des ressources naturelles? ECOFAC, Libreville, 2001.

CEFDHAC. Processus de Brazzaville, avant projet d'action. Conférence sur les Ecosystèmes de Forêts Denses Humides d'Afrique Centrale, inédit, 1996.

THE COURRIER $\mathrm{N}^{\circ} 175$. The sustainable development of tourism. Bruxelles, 1999.

EVERITT Richard. Privitisation and tourism in Privitisation in Tourism Conference, 15Nov. 1993, 8p. London, 1993.

KIRDA Philippe. Les activités cynégétiques dans la Province du Nord Cameroun entre 1983 et 1997. Consultation réalisée pour le compte du WWF, projet savanes soudaniennes du Nord. 38p. Garoua, 2000.

MINEF. Programme de conservation et de gestion de la biodiversité au Cameroun. Parc National de la Bénoué. Plan d'aménagement du Parc et de zone périphérique, 2002-2006. Yaoundé, 2001.

MINEF-UICN. Plan directeur d'aménagement du Parc National de Waza. Yaoundé, 1997. 
PNUD. Rapport sur le développement humain 1998. La pauvreté au Cameroun. 100p. Yaoundé, 2000.

SOURNIA Gérard. «Sous la Direction de». Les Aires Protégées d'Afrique Francophone. IEPF ACCT. Editions Jean Pierre de Monza. Paris, 1998.

UICN. Aménagement et gestion des aires protégées tropicales. Gland, Suisse, 1990.

MESMIN TCHINDJANG - Université de Yaoundé I, département de géographie, BP 30464 Yaoundé Cameroun.

SAMUEL AIMÉ ABOSSOLO - Université de Yaoundé I, département de géographie, BP 30464 Yaoundé Cameroun. JOSEPH ARMATHÉE - Ministère de l'environnement et de la protection de la nature.

VINCENT FRANCIS MENGA - Université de Yaoundé I, département de géographie, BP 755 Yaoundé Cameroun.

Recebido para publicação em outubro de 2006 Aceito para publicação em novembro de 2006 